\title{
Wave sensitivity analysis for periodic and arbitrarily complex composite structures
}

\author{
D. Chronopoulos ${ }^{\mathrm{a}}$, M. Collet ${ }^{\mathrm{b}}$, M. Ichchou ${ }^{\mathrm{c}}$ \\ ${ }^{a}$ Institute for Aerospace Technology \& The Composites Group, The University of \\ Nottingham, NGr 2RD, UK \\ ${ }^{b}$ LTDS, UMR-CNRS 5513, 36 Avenue Guy de Collongue, 69130 Ecully, France \\ ${ }^{c}$ Ecole Centrale de Lyon, 36 Avenue Guy de Collongue, 69130 Ecully, France
}

\begin{abstract}
Purpose: This paper presents the development of a numerical continuumdiscrete approach for computing the sensitivity of the waves propagating in periodic composite structures. The work can be directly employed for evaluating the sensitivity of the structural dynamic performance with respect to geometric and layering structural modifications.
\end{abstract}

Design/methodology/approach: A structure of arbitrary layering and geometric complexity is modelled using solid FE. A generic expression for computing the variation of the mass and the stiffness matrices of the structure with respect to the material and geometric characteristics is hereby given. The sensitivity of the structural wave properties can thus be numerically determined by computing the variability of the corresponding eigenvalues for the resulting eigenproblem. The exhibited approach is validated against the FD method as well as analytical results.

Findings: An intense wavenumber dependence is observed for the sensitivity

Email address: Dimitrios.Chronopoulos@nottingham.ac.uk (D. Chronopoulos) 
results of the sandwich structure. This exhibits the importance and potential of the presented tool with regard to the optimization of layered structures for specific applications. The model can also be used for computing the effect of the inclusion of smart layers such as auxetics and piezoelectrics.

Originality/value: The paper presents the first continuum-discrete approach specifically developed for accurately and efficiently computing the sensitivity of the wave propagation data for periodic composite structures irrespective of their size. The considered structure can be of arbitrary layering and material characteristics as FE modelling is employed.

Keywords: Sensitivity analysis, Wave propagation, Composite structures

\section{Introduction}

Layered and complex structures are nowadays widely used within the aerospace, automotive, construction and energy sectors with a general increase tendency. The wave propagation data for such structures are often employed for energy harvesting, vibration control, health monitoring and vibroacoustic transmission modelling purposes. Optimizing the layer characteristics of such structures for certain objectives is often a challenging task due to the large number of varying parameters to be considered as well as due to the lack of exact modelling approaches. The same it true about taking into account for the effect of these parametric uncertainties on the structural behaviour.

The numerical analysis of wave propagation within periodic structures was firstly considered in the pioneering work of the author of [1]. The work was extended to two dimensional media in [2]. In [3] the authors proposed 
a structured linearization method using state space eigenvalue problem for large matrices among other considerations for smoothening the solution of wave propagation in periodic structures. The WFE method was introduced in $[4,5]$ in order to facilitate the post-processing of the eigenproblem solutions and further improve the computational efficiency of the method. The vibration of a uniform waveguide using the same technique was investigated by the authors of $[6,7,8]$. The WFE method for two dimensional structures was introduced in [9] . The same method was used in [10] in order to compute the dynamic response of two dimensional infinite structures. The WFE has recently found applications in predicting the vibroacoustic and dynamic performance of composite panels and shells $[11,12,13,14,15,16,17]$, with pressurized shells $[18,19]$ and complex periodic structures [20, 21, 22] having been investigated. The variability of acoustic transmission through layered structures $[23,24]$, as well as wave steering effects in anisotropic composites [25] have been modelled through the same methodology.

Structural sensitivity analysis is of great importance for understanding the overall impact of a design parameter variation to the structural performance which is to be optimised. Accurate sensitivity models are an important tool for design optimization, system identification as well as for statistical mechanics analysis. Many authors [26, 27, 28, 29] have been focusing on the eigenvalue derivative analysis of a structural system. With regard to the variability analysis of the waves travelling within a structural medium the conducted work has been mainly focused on deriving expressions [30, 31] of the stochastic wave parameters from analytical models. In [32] the authors conduct a design sensitivity analysis by a wave based approach. Considering 
numerical approaches the authors in [33] used Bloch's theorem in conjunction with the FE method in order to calculate the sensitivity of the acoustic waves within an auxetic honeycomb, while with regard to the computation of the variability of the propagating waves the authors in $[34,35]$ have presented a stochastic WFE method approach for computing the stochastic wave propagation in one dimensional media.

In this paper a continuum-discrete approach for efficiently computing the sensitivity of the wave propagation data for periodic structures is presented. The considered structure can be of arbitrary layering and material characteristics as FE modelling is employed. The effect of local parameter variation (e.g. varying the stiffener thickness or adding mass to a single location) can also be considered. The sensitivities of the mass and stiffness matrices for a solid FE are computed with respect to any structural parameter including the material characteristics and the thickness of the element. The sensitivity of the propagating structural waves can thus be numerically determined. The exhibited approach is validated against the FD method as well as analytical results.

The paper is organized as follows: In Sec.2 the formulation of the sensitivity of the waves propagating within the periodic structure is elaborated. More precisely in Sec.2.1 a general approach for two dimensional waveguides is adopted while in Sec.2.2 a more efficient approach for one dimensional waveguides is considered. In Sec.3 the method is validated by comparison to analytical results for a metallic waveguide as well as by comparison to a FD approach for a layered sandwich waveguide. Conclusions on the presented work are given in Sec.4, while the formulation of the generic expressions for 
the stiffness and mass matrices of a solid FE are presented in the Appendix A.

\section{Sensitivity of the wave propagation data}

\subsection{General formulation}

\subsubsection{Formulation of the PST for an arbitrary structural segment}

A periodic segment of a panel having arbitrary layering and complexity is hereby considered (see Fig.1) with $l_{x}, l_{y}$ its dimensions in the $x$ and $y$ directions respectively. The segment is modelled using a conventional FE software. The mass and stiffness matrices of the segment $\mathbb{M}$ and $\mathbb{K}$ are extracted and the DoF set $\mathbf{q}$ is reordered according to a predefined sequence such as:

$$
\mathbf{q}=\left\{\begin{array}{lllllllll}
\mathbf{q}_{I} & \mathbf{q}_{B} & \mathbf{q}_{T} & \mathbf{q}_{L} & \mathbf{q}_{R} & \mathbf{q}_{L B} & \mathbf{q}_{R B} & \mathbf{q}_{L T} & \mathbf{q}_{R T}
\end{array}\right\}^{\top}
$$

corresponding to the internal, the interface edge and the interface corner DoF (see Fig.1). The free harmonic vibration equation of motion for the modelled segment is written as:

$$
\left[\mathbb{K}-\omega^{2} \mathbb{M}\right] \mathbf{q}=\mathbf{0}
$$

The analysis then follows as in [36] with the following relations being assumed for the displacement DoF under the passage of a time-harmonic wave:

$$
\begin{gathered}
\mathbf{q}_{\mathbf{R}}=\mathrm{e}^{-i \varepsilon_{x}} \mathbf{q}_{\mathbf{L}}, \quad \mathbf{q}_{\mathbf{T}}=\mathrm{e}^{-i \varepsilon_{y}} \mathbf{q}_{\mathbf{B}} \\
\mathbf{q}_{\mathbf{R B}}=\mathrm{e}^{-i \varepsilon_{x}} \mathbf{q}_{\mathbf{L B}}, \quad \mathbf{q}_{\mathbf{L T}}=\mathrm{e}^{-i \varepsilon_{y}} \mathbf{q}_{\mathbf{L B}}, \quad \mathbf{q}_{\mathbf{R T}}=\mathrm{e}^{-i \varepsilon_{x}-i \varepsilon_{y}} \mathbf{q}_{\mathbf{L B}}
\end{gathered}
$$

with $\varepsilon_{x}$ and $\varepsilon_{y}$ the propagation constants in the $x$ and $y$ directions related to the phase difference between the sets of DoF. The wavenumbers $k_{x}, k_{y}$ are directly related to the propagation constants through the relation:

$$
\varepsilon_{x}=k_{x} L_{x}, \quad \varepsilon_{y}=k_{y} L_{y}
$$




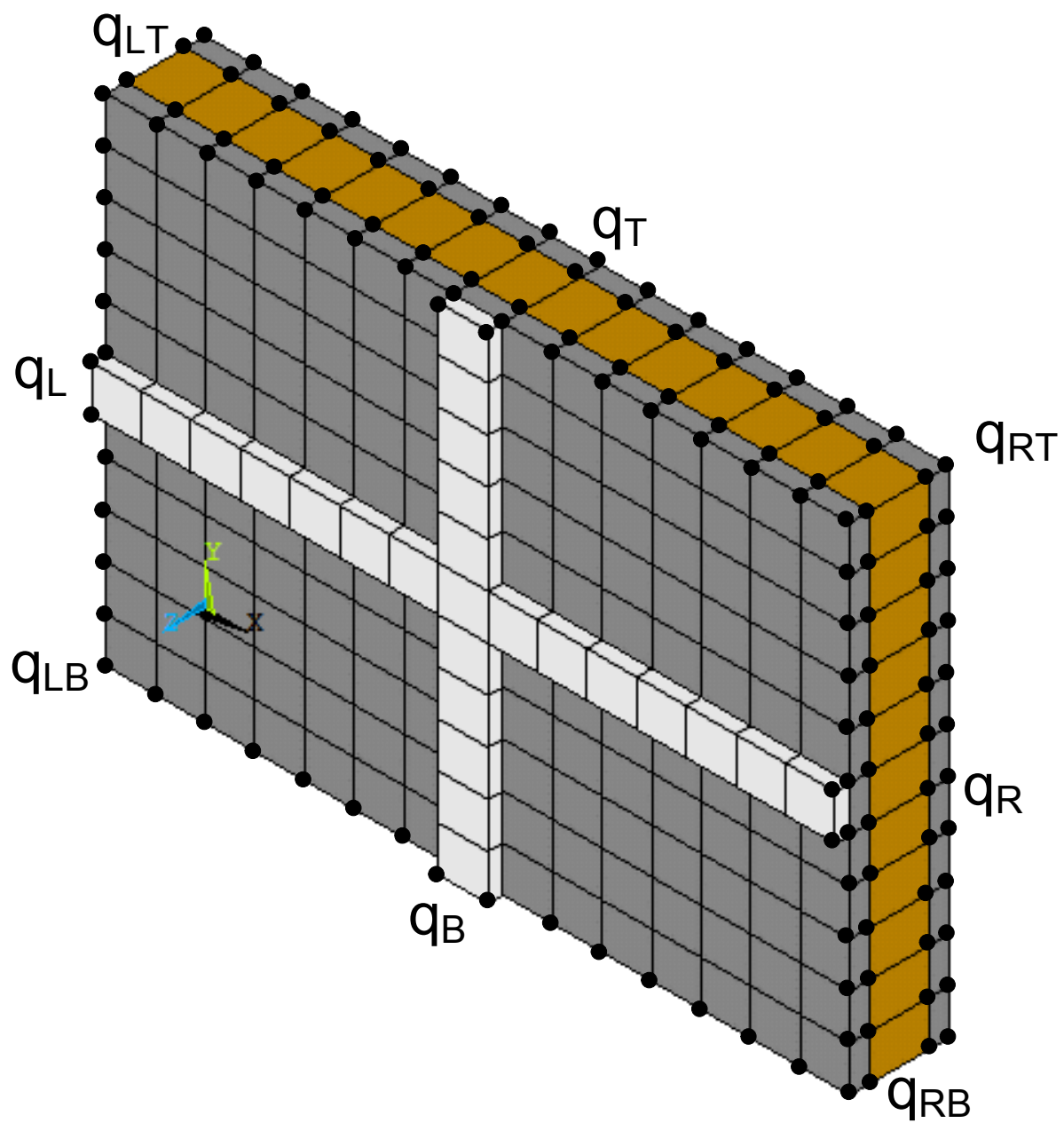

Figure 1: A composite panel modelled using PST, assuming wave propagation in $x$ direction 
Considering Eq.(3) in tensorial form gives:

$$
\mathbf{q}=\left[\begin{array}{cccc}
\mathbf{I} & \mathbf{0} & \mathbf{0} & \mathbf{0} \\
\mathbf{0} & \mathbf{I} & \mathbf{0} & \mathbf{0} \\
\mathbf{0} & \mathbf{I} \mathrm{e}^{-i \varepsilon_{y}} & \mathbf{0} & \mathbf{0} \\
\mathbf{0} & \mathbf{0} & \mathbf{I} & \mathbf{0} \\
\mathbf{0} & \mathbf{0} & \mathbf{I} \mathrm{e}^{-i \varepsilon_{x}} & \mathbf{0} \\
\mathbf{0} & \mathbf{0} & \mathbf{0} & \mathbf{I} \\
\mathbf{0} & \mathbf{0} & \mathbf{0} & \mathbf{I} \mathrm{e}^{-i \varepsilon_{x}} \\
\mathbf{0} & \mathbf{0} & \mathbf{0} & \mathbf{I}^{-i \varepsilon_{y}} \\
\mathbf{0} & \mathbf{0} & \mathbf{0} & \mathbf{I}^{-i \varepsilon_{x}-i \varepsilon_{y}}
\end{array}\right] \mathbf{x}=\mathbf{R x}
$$

with $\mathbf{x}$ the reduced set of DoF: $\mathbf{x}=\left\{\begin{array}{llll}\mathbf{q}_{\mathbf{I}} & \mathbf{q}_{\mathbf{B}} & \mathbf{q}_{\mathbf{L}} & \mathbf{q}_{\mathbf{L B}}\end{array}\right\}^{\top}$. The equation of free harmonic vibration of the modelled segment can now be written as:

$$
\left[\mathbf{R}^{*} \mathbb{K} \mathbf{R}-\omega^{2} \mathbf{R}^{*} \mathbb{M} \mathbf{R}\right] \mathbf{x}=\mathbf{0}
$$

with ${ }^{*}$ denoting the Hermitian transpose. The most practical procedure for extracting the wave propagation characteristics of the segment from Eq.(6) is injecting a set of assumed propagation constants $\varepsilon_{x}, \varepsilon_{y}$. The set of these constants can be chosen in relation to the direction of propagation towards which the wavenumbers are to be sought and according to the desired resolution of the wavenumber curves. Eq.(6) is then transformed into a standard eigenvalue problem and can be solved for the eigenvector $\mathbf{x}$ which describe the deformation of the segment under the passage of each wave type at an angular frequency equal to the square root of the corresponding eigenvalue $\lambda=\omega^{2}$. 
A complete description of each passing wave including its $x$ and $y$ directional wavenumbers and its wave shape for a certain frequency is therefore acquired. It is noted that the periodicity condition is defined modulo $2 \pi$, therefore solving Eq.(6) with a set of $\varepsilon_{x}, \varepsilon_{y}$ varying from 0 to $2 \pi$ will suffice for capturing the entirety of the structural waves. Further considerations on reducing the computational expense of the problem are discussed in [36]. It should be noted that only propagating waves will be considered in the subsequent analysis. Evanescent waves can also be captured by introducing imaginary values for $\varepsilon_{x}, \varepsilon_{y}$ however the precise computation of these waves would require a very fine resolution of the propagation constants, drastically increasing the computational effort.

\subsubsection{Parametric sensitivity}

It is noted that matrices $\mathbf{K}=\mathbf{R}^{*} \mathbb{K} \mathbf{R}$ and $\mathbf{M}=\mathbf{R}^{*} \mathbf{M} \mathbf{R}$ in Eq.(6) are Hermitian therefore their resulting eigenvalues are real and the eigenvectors will be orthogonal. Assuming the known eigenvalue $\lambda_{0 i}$ and the corresponding eigenvector $\mathbf{x}_{0 i}$ for the problem described in Eq.(6), then the following is true

$$
\mathbf{K}_{0} \mathbf{x}_{0 i}=\lambda_{0 i} \mathbf{M}_{0} \mathbf{x}_{0 i}
$$

Now if matrices $\mathbf{K}_{0}, \mathbf{M}_{0}$ are changed by a small amount, say $\delta \mathbf{K}, \delta \mathbf{M}$ then the eigenvalue $\lambda_{0 i}$ and the corresponding eigenvector $\mathbf{x}_{0 i}$ will also be perturbed so that

$$
\begin{gathered}
\mathbf{K}=\mathbf{K}_{0}+\delta \mathbf{K} \\
\mathbf{M}=\mathbf{M}_{0}+\delta \mathbf{M} \\
\lambda_{i}=\lambda_{0 i}+\delta \lambda_{i} \\
\mathbf{x}_{i}=\mathbf{x}_{0 i}+\delta \mathbf{x}_{i}
\end{gathered}
$$


A direct consequence of the orthogonality of the eigenvectors is that

$$
\mathbf{x}_{0 k}^{\top} \mathbf{M}_{0} \mathbf{x}_{0 i}=\delta_{i}^{k}
$$

with $\delta_{i}^{k}$ the Kronecker delta function. Substituting Eq.(8) into Eq.(7), we get

$$
\left(\mathbf{K}_{0}+\delta \mathbf{K}\right)\left(\mathbf{x}_{0 i}+\delta \mathbf{x}_{i}\right)=\left(\lambda_{0 i}+\delta \lambda_{i}\right)\left(\mathbf{M}_{0}+\delta \mathbf{M}\right)\left(\mathbf{x}_{0 i}+\delta \mathbf{x}_{i}\right)
$$

then expanding and using Eq.(7) we can write

$$
\begin{aligned}
& \delta \mathbf{K x}_{0 i}+\mathbf{K}_{0} \delta \mathbf{x}_{i}+\delta \mathbf{K} \delta \mathbf{x}_{i}= \\
& \lambda_{0 i} \mathbf{M}_{0} \delta \mathbf{x}_{i}+\lambda_{0 i} \delta \mathbf{M} \mathbf{x}_{0 i}+\delta \lambda_{i} \mathbf{M}_{0} \mathbf{x}_{0 i}+\lambda_{0 i} \delta \mathbf{M} \delta \mathbf{x}_{i}+\delta \lambda_{i} \delta \mathbf{M} \mathbf{x}_{0 i}+\delta \lambda_{i} \mathbf{M}_{0} \delta \mathbf{x}_{i}+\delta \lambda_{i} \delta \mathbf{M} \delta \mathbf{x}_{i}
\end{aligned}
$$

and removing the higher-order terms simplifies to

$$
\mathbf{K}_{0} \delta \mathbf{x}_{i}+\delta \mathbf{K x}_{0 i}=\lambda_{0 i} \mathbf{M}_{0} \delta \mathbf{x}_{i}+\lambda_{0 i} \delta \mathbf{M} \mathbf{x}_{0 i}+\delta \lambda_{i} \mathbf{M}_{0} \mathbf{x}_{0 i}
$$

The orthogonality properties of the unperturbed eigenvectors in Eq.(6) allow for using them as a basis for expressing the perturbed eigenvectors. That is the perturbation of the eigenvector $\mathbf{x}_{0 i}$ can be expressed as

$$
\delta \mathbf{x}_{i}=\sum_{k=1}^{N} \epsilon_{i k} \mathbf{x}_{0 k}
$$

with $\epsilon_{i k}$ small unknown constants. Substituting Eq.(13) into Eq.(12) gives

$$
\mathbf{K}_{0} \sum_{k=1}^{N} \epsilon_{i k} \mathbf{x}_{0 k}+\delta \mathbf{K} \mathbf{x}_{0 i}=\lambda_{0 i} \mathbf{M}_{0} \sum_{k=1}^{N} \epsilon_{i k} \mathbf{x}_{0 k}+\lambda_{0 i} \delta \mathbf{M x}_{0 i}+\delta \lambda_{i} \mathbf{M}_{0} \mathbf{x}_{0 i}
$$

which can also be written as

$$
\sum_{k=1}^{N} \epsilon_{i k} \mathbf{K}_{0} \mathbf{x}_{0 k}+\delta \mathbf{K x}_{0 i}=\lambda_{0 i} \mathbf{M}_{0} \sum_{k=1}^{N} \epsilon_{i k} \mathbf{x}_{0 k}+\lambda_{0 i} \delta \mathbf{M x}_{0 i}+\delta \lambda_{i} \mathbf{M}_{0} \mathbf{x}_{0 i}
$$


Making use of Eq.(7) it is true that

$$
\sum_{k=1}^{N} \epsilon_{i k} \lambda_{0 k} \mathbf{M}_{0} \mathbf{x}_{0 k}+\delta \mathbf{K} \mathbf{x}_{0 i}=\lambda_{0 i} \mathbf{M}_{0} \sum_{k=1}^{N} \epsilon_{i k} \mathbf{x}_{0 k}+\lambda_{0 i} \delta \mathbf{M} \mathbf{x}_{0 i}+\delta \lambda_{i} \mathbf{M}_{0} \mathbf{x}_{0 i}
$$

Again due to the orthogonality of the eigenvectors in Eq.(9), the summations can be removed by left multiplying by $\mathbf{x}_{0 i}^{\top}$, therefore

$$
\mathbf{x}_{0 i}^{\top} \epsilon_{i i} \lambda_{0 i} \mathbf{M}_{0} \mathbf{x}_{0 i}+\mathbf{x}_{0 i}^{\top} \delta \mathbf{K} \mathbf{x}_{0 i}=\lambda_{0 i} \mathbf{x}_{0 i}^{\top} \mathbf{M}_{0} \epsilon_{i i} \mathbf{x}_{0 i}+\lambda_{0 i} \mathbf{x}_{0 i}^{\top} \delta \mathbf{M x}_{0 i}+\delta \lambda_{i} \mathbf{x}_{0 i}^{\top} \mathbf{M}_{0} \mathbf{x}_{0 i}
$$

and eliminating the equal terms gives

$$
\mathbf{x}_{0 i}^{\top} \delta \mathbf{K} \mathbf{x}_{0 i}=\lambda_{0 i} \mathbf{x}_{0 i}^{\top} \delta \mathbf{M x}_{0 i}+\delta \lambda_{i} \mathbf{x}_{0 i}^{\top} \mathbf{M}_{0} \mathbf{x}_{0 i}
$$

Rearranging the expression with regard to $\delta \lambda_{i}$ we get

$$
\delta \lambda_{i}=\frac{\mathbf{x}_{0 i}^{\top}\left(\delta \mathbf{K}-\lambda_{0 i} \delta \mathbf{M}\right) \mathbf{x}_{0 i}}{\mathbf{x}_{0 i}^{\top} \mathbf{M}_{0} \mathbf{x}_{0 i}}
$$

However, because of Eq.(9) the expression of the eigenvalue perturbation is given as

$$
\delta \lambda_{i}=\mathbf{x}_{0 i}^{\top}\left(\delta \mathbf{K}-\lambda_{0 i} \delta \mathbf{M}\right) \mathbf{x}_{0 i}
$$

When the partial derivatives of $\mathbf{K}, \mathbf{M}$ with regard to a design parameter $\beta$ are known then the sensitivity of an eigenvalue $\lambda_{i}$ to this design parameter will be equal to

$$
\frac{\partial \lambda_{i}}{\partial \beta}=\mathbf{x}_{0 i}^{\top}\left(\frac{\partial \mathbf{K}}{\partial \beta}-\lambda_{0 i} \frac{\partial \mathbf{M}}{\partial \beta}\right) \mathbf{x}_{0 i}
$$

The global mass and stiffness matrices $\mathbb{M}, \mathbb{K}$ of the structural segment are formed by adding the local mass and stiffness matrices of individual FEs. Therefore it can be concluded that when the expression of the partial derivatives for every local mass and stiffness matrix $\frac{\partial \mathbf{m}}{\partial \beta}, \frac{\partial \mathbf{k}}{\partial \beta}$ are known then 
the expressions for the global matrices $\frac{\partial \mathbb{M}}{\partial \beta}, \frac{\partial \mathbb{K}}{\partial \beta}$ can be derived simply by adding the expressions of the local matrices together. Eq.(21) can therefore be written as:

$$
\frac{\partial \lambda_{i}}{\partial \beta}=\mathbf{x}_{0 i}^{\top}\left(\mathbf{R}^{*} \frac{\partial \mathbb{K}}{\partial \beta} \mathbf{R}-\lambda_{0 i} \mathbf{R}^{*} \frac{\partial \mathbb{M}}{\partial \beta} \mathbf{R}\right) \mathbf{x}_{0 i}
$$

It is known however that $\frac{\partial \lambda_{i}}{\partial \beta}=\frac{\partial\left(\omega_{i}^{2}\right)}{\partial \beta}$, therefore

$$
\frac{\partial \lambda_{i}}{\partial \beta}=\frac{\partial\left(\omega_{i}^{2}\right)}{\partial \beta}=\frac{\frac{\partial\left(\omega_{i}^{2}\right)}{\partial \omega_{i}}}{\frac{\partial \beta}{\partial \omega_{i}}}=2 \omega_{i} \frac{\partial \omega_{i}}{\partial \beta}
$$

Eq.(22) can therefore be written as:

$$
\frac{\partial \omega_{i}}{\partial \beta}=\frac{\mathbf{x}_{0 i}^{\top}\left(\mathbf{R}^{*} \frac{\partial \mathbb{K}}{\partial \beta} \mathbf{R}-\lambda_{0 i} \mathbf{R}^{*} \frac{\partial \mathbb{M}}{\partial \beta} \mathbf{R}\right) \mathbf{x}_{0 i}}{2 \omega_{i}}
$$

with $\omega_{i}$ the angular frequency at which the set of $\varepsilon_{x}, \varepsilon_{y}$ is true for the $i$ wave type described by the $\mathbf{x}_{0 i}$ deformation. The generic symbolic expressions of the $\mathbf{m}, \mathbf{k}$ matrices for an orthotropic structural segment modelled with a linear solid FE is given in Appendix A. The wavenumber sensitivity $\frac{\partial k_{i}}{\partial \beta}$ can be deduced as

$$
\frac{\partial k_{i}}{\partial \beta}=-\frac{\partial k_{i}}{\partial \omega_{i}} \frac{\partial \omega_{i}}{\partial \beta}=-\frac{1}{c_{g, i}} \frac{\partial \omega_{i}}{\partial \beta}
$$

with $c_{g, i}=\frac{\partial \omega_{i}}{\partial k_{i}}$ the group velocity associated with the wave type $i$ at frequency $\omega_{i}$ which can be evaluated by avoiding any numerical differentiation as exhibited in [37].

\subsection{Transfer matrix formulation}

\subsubsection{Condensation process}

When wave propagation is considered only in the $x$ direction, the problem can be condensed using a transfer matrix approach as in [4]. The DSM of the 
structural segment is again partitioned, this time with regard to its left/right sides and internal DoF

$$
\left[\begin{array}{ccc}
\mathbf{D}_{L L} & \mathbf{D}_{L I} & \mathbf{D}_{L R} \\
\mathbf{D}_{I L} & \mathbf{D}_{I I} & \mathbf{D}_{I R} \\
\mathbf{D}_{R L} & \mathbf{D}_{R I} & \mathbf{D}_{R R}
\end{array}\right]\left\{\begin{array}{c}
\mathbf{q}_{L} \\
\mathbf{q}_{I} \\
\mathbf{q}_{R}
\end{array}\right\}=\left\{\begin{array}{c}
\mathbf{f}_{L} \\
\mathbf{0} \\
\mathbf{f}_{R}
\end{array}\right\}
$$

with $\mathbf{q}$ the displacement and $\mathbf{f}$ the force vectors. Using a Guyan type condensation for the internal DoF the problem can be expressed as

$$
\left[\begin{array}{cc}
\mathbf{D}_{L L}-\mathbf{D}_{L I} \mathbf{D}_{I I}^{-1} \mathbf{D}_{I L} & \mathbf{D}_{L R}-\mathbf{D}_{L I} \mathbf{D}_{I I}^{-1} \mathbf{D}_{I R} \\
\mathbf{D}_{R L}-\mathbf{D}_{R I} \mathbf{D}_{I I}^{-1} \mathbf{D}_{I L} & \mathbf{D}_{R R}-\mathbf{D}_{R I} \mathbf{D}_{I I}^{-1} \mathbf{D}_{I R}
\end{array}\right]\left\{\begin{array}{c}
\mathbf{q}_{L} \\
\mathbf{q}_{R}
\end{array}\right\}=\left\{\begin{array}{c}
\mathbf{f}_{L} \\
\mathbf{f}_{R}
\end{array}\right\}
$$

Assuming that no external forces are applied on the segment the displacement continuity and force equilibrium equations at the interface of two consecutive periodic segments $s$ and $s+1$ give

$$
\begin{gathered}
\mathbf{q}_{L}^{s+1}=\mathbf{q}_{R}^{s} \\
\mathbf{f}_{L}^{s+1}=-\mathbf{f}_{R}^{s}
\end{gathered}
$$

Using Eqs.(27),(28) the relation of the displacements and forces of the left and right sides of the segment can be written as

$$
\left\{\begin{array}{l}
\mathbf{q}_{L}^{s+1} \\
\mathbf{f}_{L}^{s+1}
\end{array}\right\}=\mathbf{T}\left\{\begin{array}{c}
\mathbf{q}_{L}^{s} \\
\mathbf{f}_{L}^{s}
\end{array}\right\}
$$

and the expression of the symplectic transfer matrix $\mathbf{T}$ can be written as

$$
\mathbf{T}=\left[\begin{array}{ll}
\mathbf{D}_{11} & \mathbf{D}_{12} \\
\mathbf{D}_{21} & \mathbf{D}_{22}
\end{array}\right]
$$


with

$$
\begin{aligned}
& \mathbf{D}_{11}=-\left(\mathbf{D}_{L R}-\mathbf{D}_{L I} \mathbf{D}_{I I}^{-1} \mathbf{D}_{I R}\right)^{-1}\left(\mathbf{D}_{L L}-\mathbf{D}_{L I} \mathbf{D}_{I I}^{-1} \mathbf{D}_{I L}\right) \\
& \mathbf{D}_{12}=\left(\mathbf{D}_{L R}-\mathbf{D}_{L I} \mathbf{D}_{I I}^{-1} \mathbf{D}_{I R}\right)^{-1} \\
& \mathbf{D}_{21}=-\mathbf{D}_{R L}+\mathbf{D}_{R I} \mathbf{D}_{I I}^{-1} \mathbf{D}_{I L}+\left(\mathbf{D}_{R R}-\mathbf{D}_{R I} \mathbf{D}_{I I}^{-1} \mathbf{D}_{I R}\right)\left(\mathbf{D}_{L R}-\mathbf{D}_{L I} \mathbf{D}_{I I}^{-1} \mathbf{D}_{I R}\right)^{-1}\left(\mathbf{D}_{L L}-\mathbf{D}_{L I} \mathbf{D}_{I I}^{-1} \mathbf{D}_{I L}\right. \\
& \mathbf{D}_{22}=-\left(\mathbf{D}_{R R}-\mathbf{D}_{R I} \mathbf{D}_{I I}^{-1} \mathbf{D}_{I R}\right)\left(\mathbf{D}_{L R}-\mathbf{D}_{L I} \mathbf{D}_{I I}^{-1} \mathbf{D}_{I R}\right)^{-1}
\end{aligned}
$$

Free wave propagation is described by the eigenproblem

$$
\gamma\left\{\begin{array}{c}
\mathbf{q}_{L}^{s} \\
\mathbf{f}_{L}^{s}
\end{array}\right\}=\mathbf{T}\left\{\begin{array}{c}
\mathbf{q}_{L}^{s} \\
\mathbf{f}_{L}^{s}
\end{array}\right\}
$$

whose solution $\gamma_{i}$ is related to the structural wavenumber $k_{x i}$ by

$$
k_{x i}=-\frac{\ln \left(\gamma_{i}\right)}{\mathrm{i} l_{x}}
$$

\subsubsection{Parametric sensitivity}

Assuming the known eigenvalue $\gamma_{0 i}$ and the corresponding eigenvector $\mathbf{z}_{0 i}=\left\{\begin{array}{c}\mathbf{q}_{0 i} \\ \mathbf{f}_{0 i}\end{array}\right\}$ for the problem described in Eq.(32), then the following is true

$$
\gamma_{0 i} \mathbf{z}_{0 i}=\mathbf{T}_{0} \mathbf{z}_{0 i}
$$

Now if the matrix $\mathbf{T}_{0}$ is changed by a small amount, say $\delta \mathbf{T}$ then the eigenvalue $\gamma_{0 i}$ and the corresponding eigenvector $\mathbf{z}_{0 i}$ will also be perturbed so that

$$
\left(\gamma_{0 i}+\delta \gamma_{i}\right)\left(\mathbf{z}_{0 i}+\delta \mathbf{z}_{i}\right)=\left(\mathbf{T}_{0}+\delta \mathbf{T}\right)\left(\mathbf{z}_{0 i}+\delta \mathbf{z}_{i}\right)
$$

Expanding we have

$$
\gamma_{0 i} \mathbf{z}_{0 i}+\gamma_{0 i} \delta \mathbf{z}_{i}+\delta \gamma_{i} \mathbf{z}_{0 i}+\delta \gamma_{i} \delta \mathbf{z}_{i}=\mathbf{T}_{0} \mathbf{z}_{0 i}+\mathbf{T}_{0} \delta \mathbf{z}_{i}+\delta \mathbf{T} \mathbf{z}_{0 i}+\delta \mathbf{T} \delta \mathbf{z}_{i}
$$


However using Eq.(34) and removing the higher-order terms eventually simplifies the relation to

$$
\gamma_{0 i} \delta \mathbf{z}_{i}+\delta \gamma_{i} \mathbf{z}_{0 i}=\mathbf{T}_{0} \delta \mathbf{z}_{i}+\delta \mathbf{T} \mathbf{z}_{0 i}
$$

The symplectic orthogonality properties [5] of the unperturbed eigenvectors of $\mathbf{T}_{0}$ suggest that if $\gamma_{i}$ is an eigenvalue of $\mathbf{T}_{0}$ with the corresponding right eigenvector $\mathbf{z}_{i}$, then $1 / \gamma_{i}$ will also be an eigenvalue of $\mathbf{T}_{0}$ with a corresponding left eigenvector $\mathbf{y}_{i}=\mathbf{z}_{i} \mathbf{J}_{n}$, with $\mathbf{J}_{n}$ a $2 n \times 2 n$ matrix given as $\mathbf{J}_{n}=\left[\begin{array}{cc}\mathbf{0} & \mathbf{I}_{n} \\ -\mathbf{I}_{n} & \mathbf{0}\end{array}\right]$. The following will also apply if the eigenvectors are appropriately scaled

$$
\mathbf{Y}_{0}^{\top} \mathbf{Z}_{0}=\mathbf{I}
$$

and

$$
\mathbf{Y}_{0}^{\top} \mathbf{T}_{0} \mathbf{Z}_{0}=\Gamma_{\mathbf{0}}
$$

with $\boldsymbol{\Gamma}_{\mathbf{0}}$ the diagonal matrix containing the eigenvalues of $\mathbf{T}_{0}$, while

$$
\mathbf{Y}_{0}=\left[\mathbf{y}_{01} \mathbf{y}_{02} \cdots \mathbf{y}_{0 n}\right]
$$

is the matrix containing the left eigenvectors of $\mathbf{T}_{0}$ and

$$
\mathbf{Z}_{0}=\left[\mathbf{z}_{01} \mathbf{z}_{02} \cdots \mathbf{z}_{0 n}\right]
$$

the matrix containing the right eigenvectors of $\mathbf{T}_{0}$. The orthogonality property allows for expressing the perturbation of the eigenvector $\mathbf{z}_{0 i}$ as

$$
\delta \mathbf{z}_{i}=\sum_{k=1}^{N} \epsilon_{i k} \mathbf{z}_{0 k}
$$


with $\epsilon_{i k}$ small unknown constants. Substituting Eq.(42) into Eq.(37) and making use of Eq.(34) once again gives

$$
\gamma_{0 i} \sum_{k=1}^{N} \epsilon_{i k} \mathbf{z}_{0 k}+\delta \gamma_{i} \mathbf{z}_{0 i}=\sum_{k=1}^{N} \epsilon_{i k} \gamma_{0 k} \mathbf{z}_{0 k}+\delta \mathbf{T} \mathbf{z}_{0 i}
$$

and due to Eq.(38), the summations can be removed by left multiplying by $\mathbf{y}_{0 i}^{\top}$, therefore

$$
\mathbf{y}_{0 i}^{\top} \epsilon_{i i} \gamma_{0 i} \mathbf{z}_{0 i}+\mathbf{y}_{0 i}^{\top} \delta \gamma_{i} \mathbf{z}_{0 i}=\mathbf{y}_{0 i}^{\top} \epsilon_{i i} \gamma_{0 i} \mathbf{z}_{0 i}+\mathbf{y}_{0 i}^{\top} \delta \mathbf{T} \mathbf{z}_{0 i}
$$

Eliminating the equal terms gives

$$
\mathbf{y}_{0 i}^{\top} \delta \gamma_{i} \mathbf{z}_{0 i}=\mathbf{y}_{0 i}^{\top} \delta \mathbf{T} \mathbf{z}_{0 i}
$$

Due to the orthogonality of the eigenvectors it is true that

$$
\mathbf{y}_{0 i}^{\top} \mathbf{z}_{0 i}=1
$$

therefore the expression of the eigenvalue perturbation $\delta \gamma_{i}$ as a function of the transfer matrix perturbation $\delta \mathbf{T}$ can be written as

$$
\delta \gamma_{i}=\mathbf{y}_{0 i}^{\top} \delta \mathbf{T} \mathbf{z}_{0 i}
$$

When the partial derivative of $\mathbf{T}$ with regard to a design parameter $\beta$ is known then the sensitivity of an eigenvalue $\gamma_{i}$ to $\beta$ is equal to

$$
\frac{\partial \gamma_{i}}{\partial \beta}=\mathbf{y}_{i}^{\top} \frac{\partial \mathbf{T}}{\partial \beta} \mathbf{z}_{i}
$$

It is known however that $\frac{\partial \gamma_{i}}{\partial \beta}=\frac{\partial\left(e^{-\mathrm{i} k_{x i} l_{x}}\right)}{\partial \beta}$, therefore

$$
\frac{\frac{\partial\left(e^{-\mathrm{i} k_{x i} l_{x}}\right)}{\partial k_{x i}}}{\frac{\partial \beta}{\partial k_{x i}}}=-\mathrm{i} l_{x} e^{-\mathrm{i} k_{x i} l_{x}} \frac{\partial k_{x i}}{\partial \beta}
$$


Eq.(48) can therefore be written as:

$$
\frac{\partial k_{x i}}{\partial \beta}=\frac{1}{-\mathrm{i} l_{x} e^{-\mathrm{i} k_{x i} l_{x}}} \mathbf{y}_{i}^{\top} \frac{\partial \mathbf{T}}{\partial \beta} \mathbf{z}_{i}
$$

The symbolic expression of the $\frac{\partial \mathbf{k}}{\partial \beta}, \frac{\partial \mathbf{m}}{\partial \beta}$ matrices for a structural segment modelled with a linear solid FE can be derived using the expressions given in Appendix A.. The corresponding expression of the DSM in Eq.(27) can therefore be directly computed. The symbolic expression of $\mathbf{T}$ as well as of the sensitivity $\frac{\partial \mathbf{T}}{\partial \beta}$ can thereafter be derived using Eq.(31). It should be noted that due to the inversion and multiplication of DSM partitions involved in Eq.(31), this approach tends to be less computationally efficient than the one presented in Sec.2.1.2, especially for large structural segments.

\section{Numerical case studies}

Two case studies are presented below in order to validate the presented work. In the first case a metallic monolayer panel is modelled by the presented approach and the results on the sensitivity of the propagating waves are compared to the values derived by the CPT. In the second case study a sandwich panel comprising facesheets of different thicknesses is modelled and the results are validated through the FD method. Both cases were modelled using the approach presented in Sec.2.1. All computations were conducted using the R2013a version of MATLAB ${ }^{\circledR}$.

\subsection{Monolayer panel}

A metallic single layer panel is hereby modelled. The panel has a thickness $h=0.003 \mathrm{~m}$ and is made of a material with a density $\rho=3000 \mathrm{~kg} / \mathrm{m}^{2}$, a 
Young's modulus $E=70 \mathrm{GPa}$ and a Poisson's ratio equal to $v=0.1$. Three equidimensional $\mathrm{FE}$ are used in order to model the structure in the sense of thickness. The first result in Fig.2 refers to the sensitivity of $\omega$ at which a specific $\varepsilon_{x}, \varepsilon_{y}$ set occurs for the first bending wave propagating in the structure. Real values are considered for $\varepsilon_{x}, \varepsilon_{y}$ throughout this paper. The result is compared to the CPT theory by differentiating the expression relating the flexural wavenumber $k_{f}$ to the angular frequency $\omega_{f}$

$$
\omega_{f}=\frac{k_{f}^{2}}{\sqrt{\frac{\rho h}{D_{m}}}}
$$

with $D_{m}=\frac{E h^{3}}{12\left(1-v^{2}\right)}$ the flexural stiffness of a monolayer panel. Very good agreement is observed between the two curves with the maximum divergence being equal to $0.18 \%$. The perturbation of $\omega$ for the first membrane wave propagating in the structure with relation to $E$ is presented in Fig.3. The result is compared to the CPT theory by differentiating the expression relating the membrane wavenumber $k_{m}$ to the angular frequency $\omega_{m}$

$$
\omega_{m}=\frac{k_{m}}{\sqrt{\frac{\rho\left(1-v^{2}\right)}{E}}}
$$

Again excellent agreement is observed between the analytical and numerical results. The perturbation of $\omega$ for the first shear wave propagating in the structure with relation to $E$ is presented in Fig.4. The result is compared to the CPT theory by differentiating the expression relating the shear wavenumber $k_{s}$ to the angular frequency $\omega_{s}$

$$
\omega_{s}=\frac{k_{s}}{\sqrt{\frac{2 \rho(1+v)}{E}}}
$$




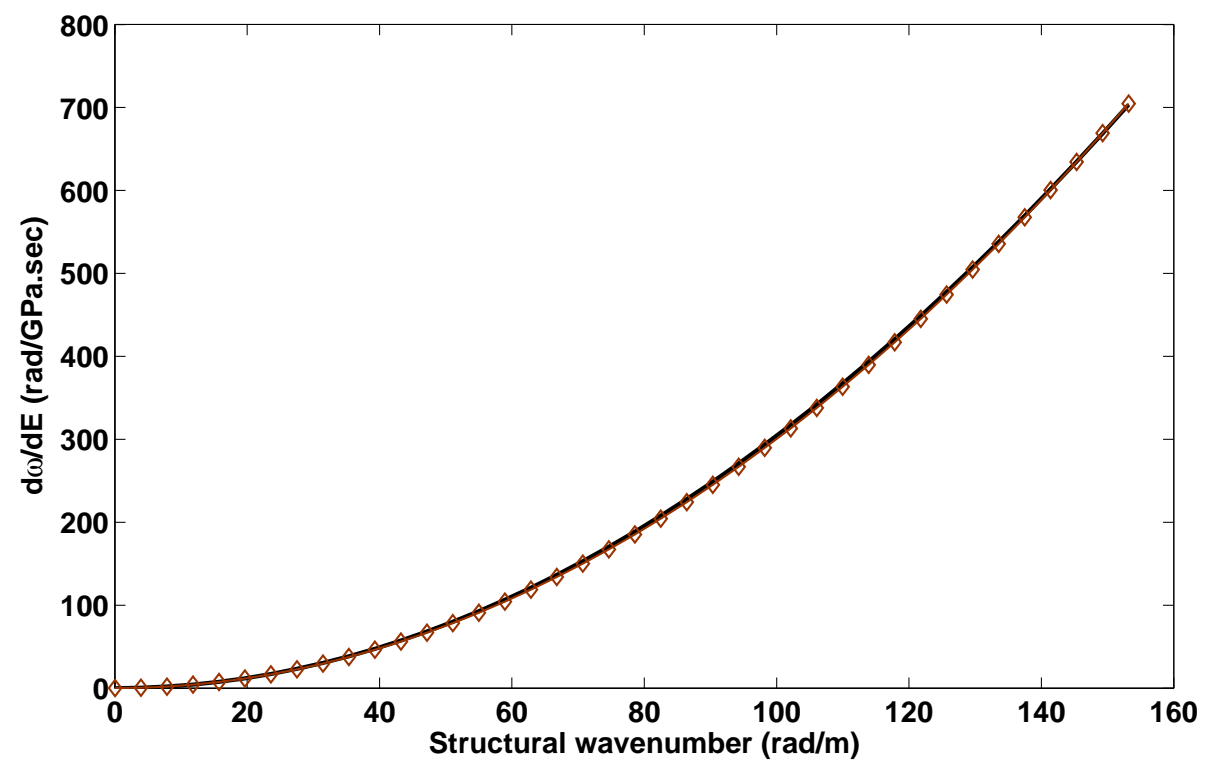

Figure 2: Sensitivity of the angular frequency $\omega$ at which the set of $\varepsilon_{x}, \varepsilon_{y}$ occur under a perturbation of $E$ for the first flexural wave type: Analytical solution $(\diamond)$, Numerical calculation $(-)$ 


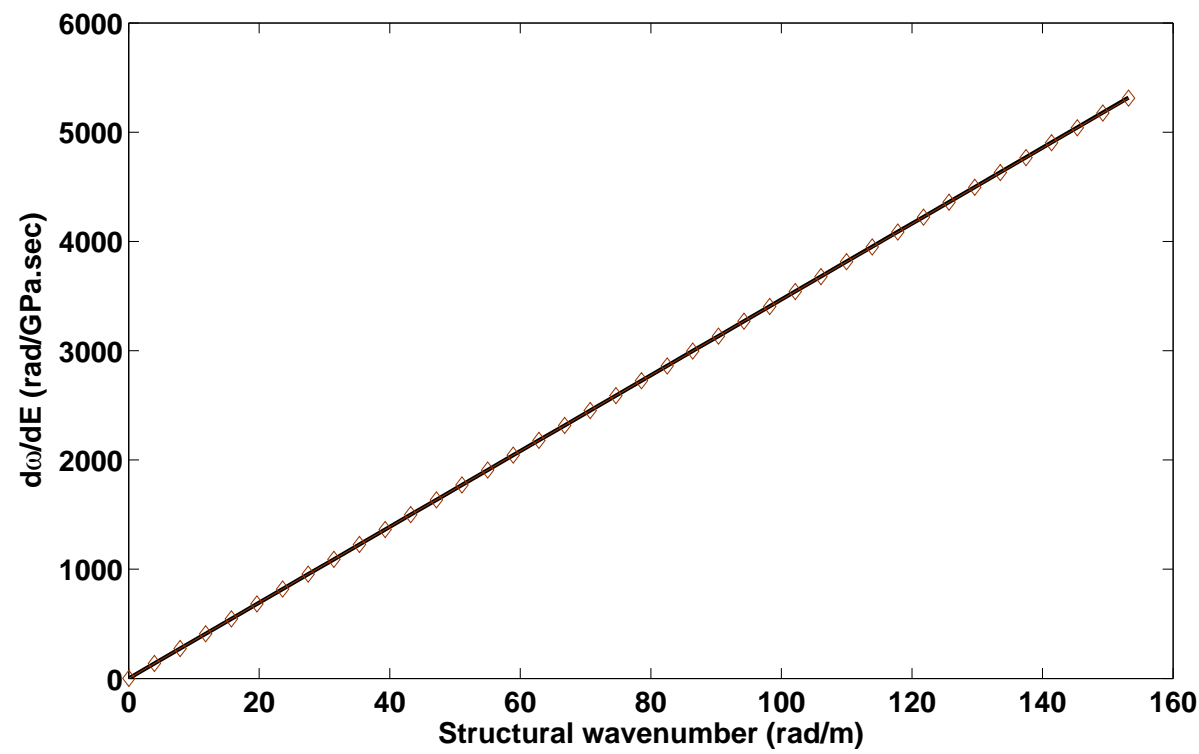

Figure 3: Sensitivity of the angular frequency $\omega$ at which the set of $\varepsilon_{x}, \varepsilon_{y}$ occur under a perturbation of $E$ for the first membrane wave type: Analytical solution ( $\diamond)$, Numerical calculation $(-)$ 


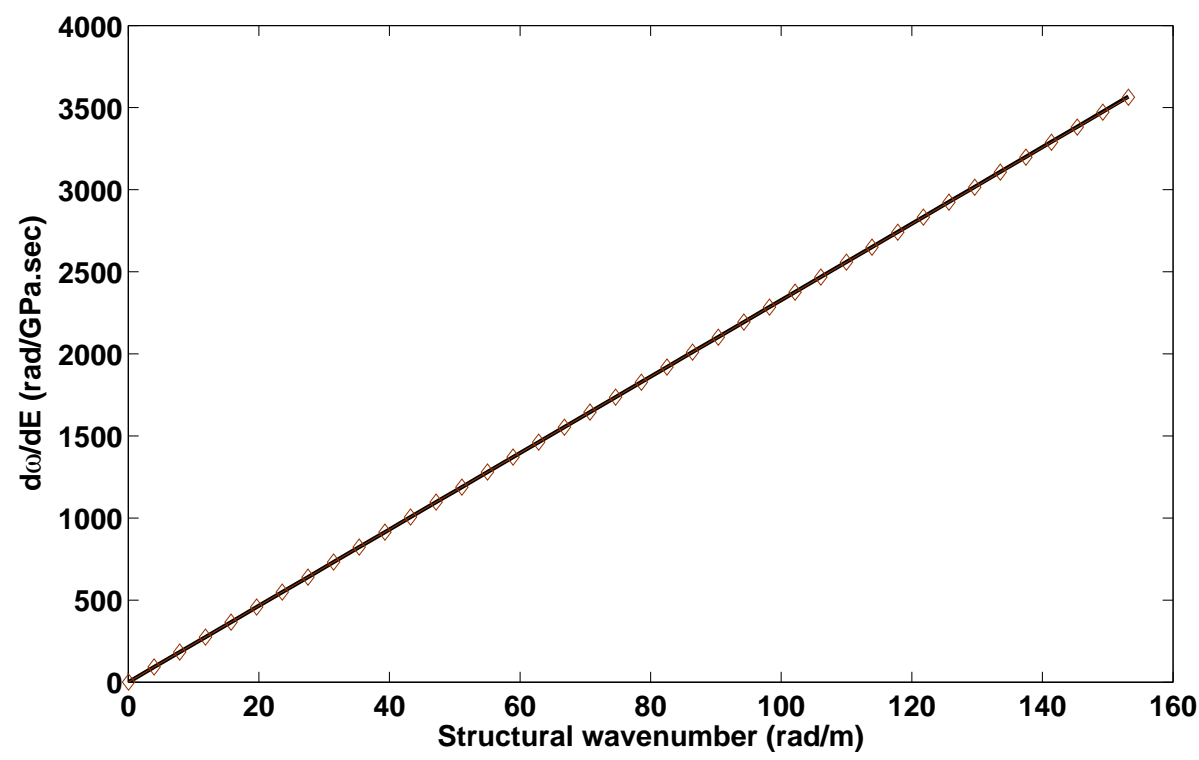

Figure 4: Sensitivity of the angular frequency $\omega$ at which the set of $\varepsilon_{x}, \varepsilon_{y}$ occur under a perturbation of $E$ for the first shear wave type: Analytical solution $(\diamond)$, Numerical calculation $(-)$

Again excellent correlation is observed between the analytical and numerical results. As expected, throughout Fig.2-4 it is exhibited that an increase of $E$ would stiffen the structure, shifting a certain wavenumber value towards higher frequencies. The perturbation of $\omega$ for the first flexural wave propagating in the structure with relation to the thickness $h$ is presented in Fig.5. Good agreement is generally observed between the results, however there is a maximum discrepancy of $4.3 \%$ between the two curves. This is probably due to an insufficiency of the analytical approach for modelling the dynamic behaviour at higher frequencies. Again an increase in thickness will stiffen the structure, shifting the same wavenumber towards higher frequencies. There is no dependence of the shear and membrane waves on the thickness of the 


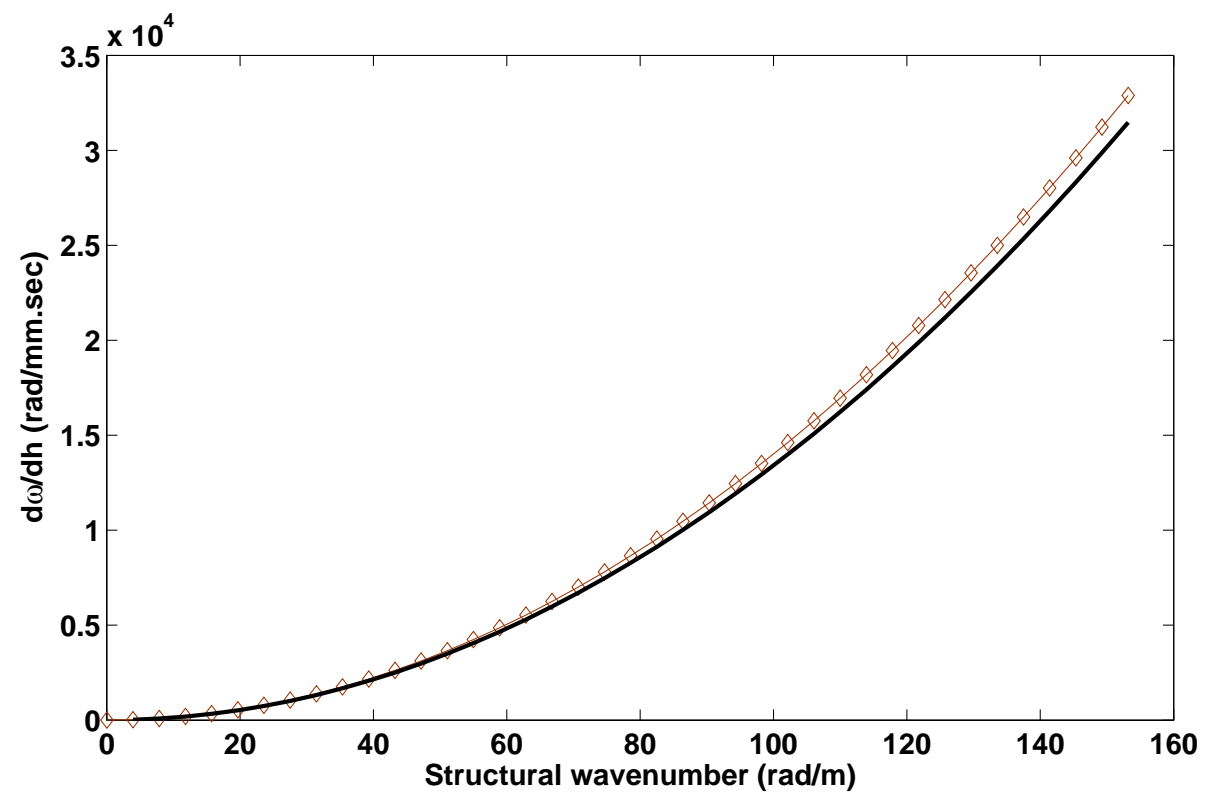

Figure 5: Sensitivity of the angular frequency $\omega$ at which the set of $\varepsilon_{x}, \varepsilon_{y}$ occur under a perturbation of the thickness of the structure for the first flexural wave type: Analytical

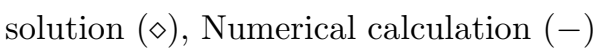

structure, therefore the corresponding results are not presented for the sake of brevity. The perturbation of $\omega$ with respect to the Poisson's ratio of the structure for the flexural, membrane and shear waves are presented in Fig.6,7 and 8 respectively. A generally good agreement is observed between the numerical and analytical results. Again for the flexural wave a maximum discrepancy of $11.5 \%$ between the two curves is taking place for high wavenumber values due to the same aforementioned reason. It is interesting to note that while increasing the Poisson's ratio of the structure acts as a stiffening effect for the flexural and membrane wave types, the same positive perturbation of $v$ will result in shifting the shear wavenumber curves towards lower frequencies. 


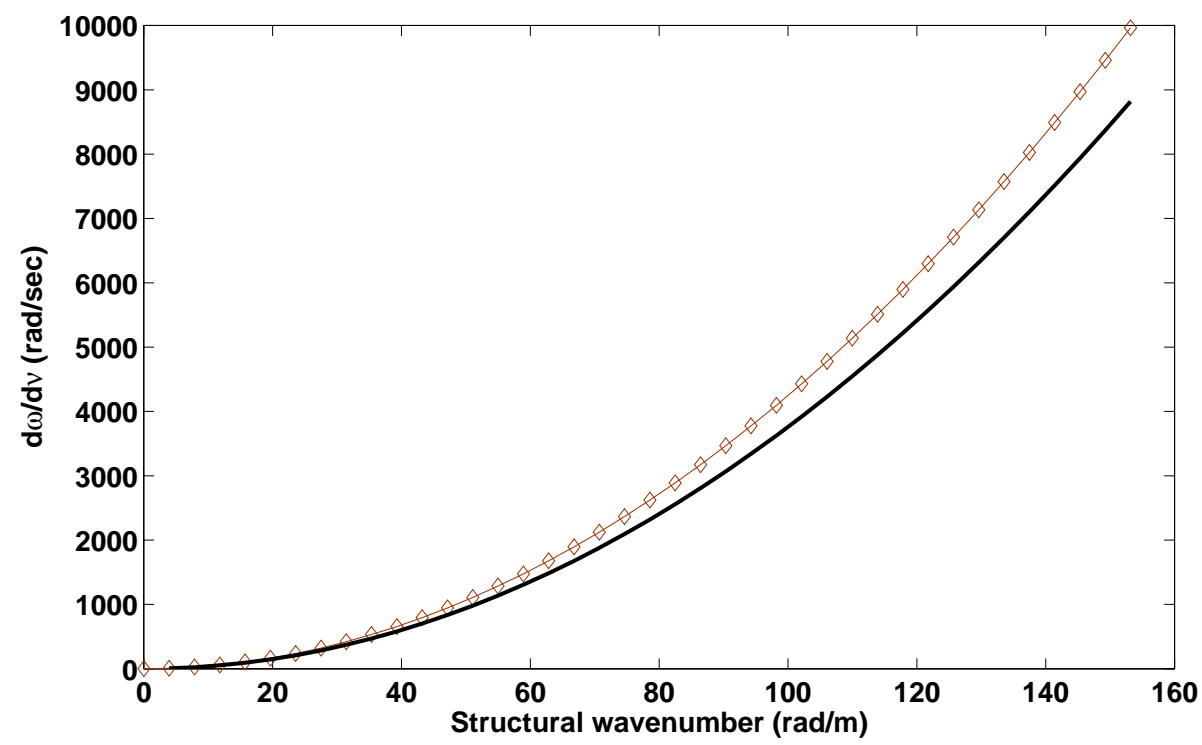

Figure 6: Sensitivity of the angular frequency $\omega$ at which the set of $\varepsilon_{x}, \varepsilon_{y}$ occur under a perturbation of the Poisson's ratio of the structure for the first flexural wave type: Analytical solution $(\diamond)$, Numerical calculation (-) 


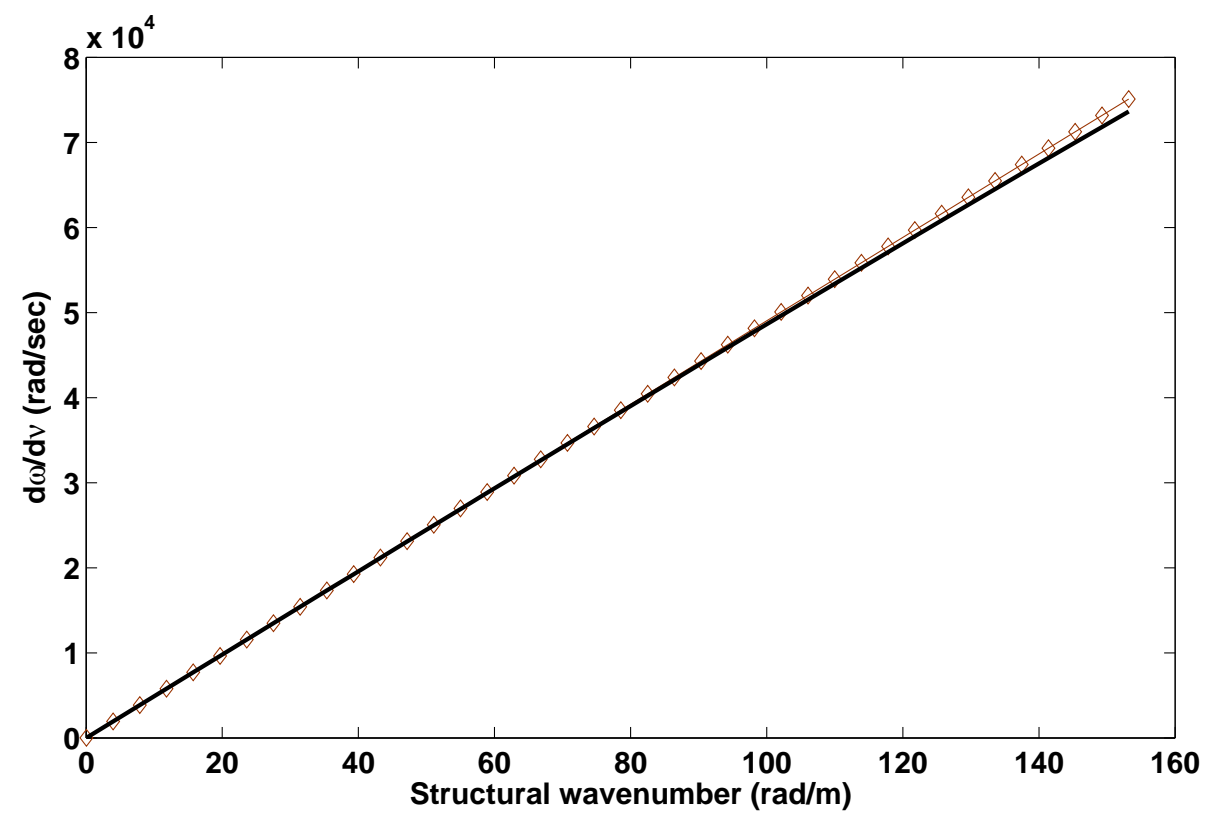

Figure 7: Sensitivity of the angular frequency $\omega$ at which the set of $\varepsilon_{x}, \varepsilon_{y}$ occur under a perturbation of the Poisson's ratio of the structure for the first membrane wave type: Analytical solution ( $\diamond)$, Numerical calculation (-) 


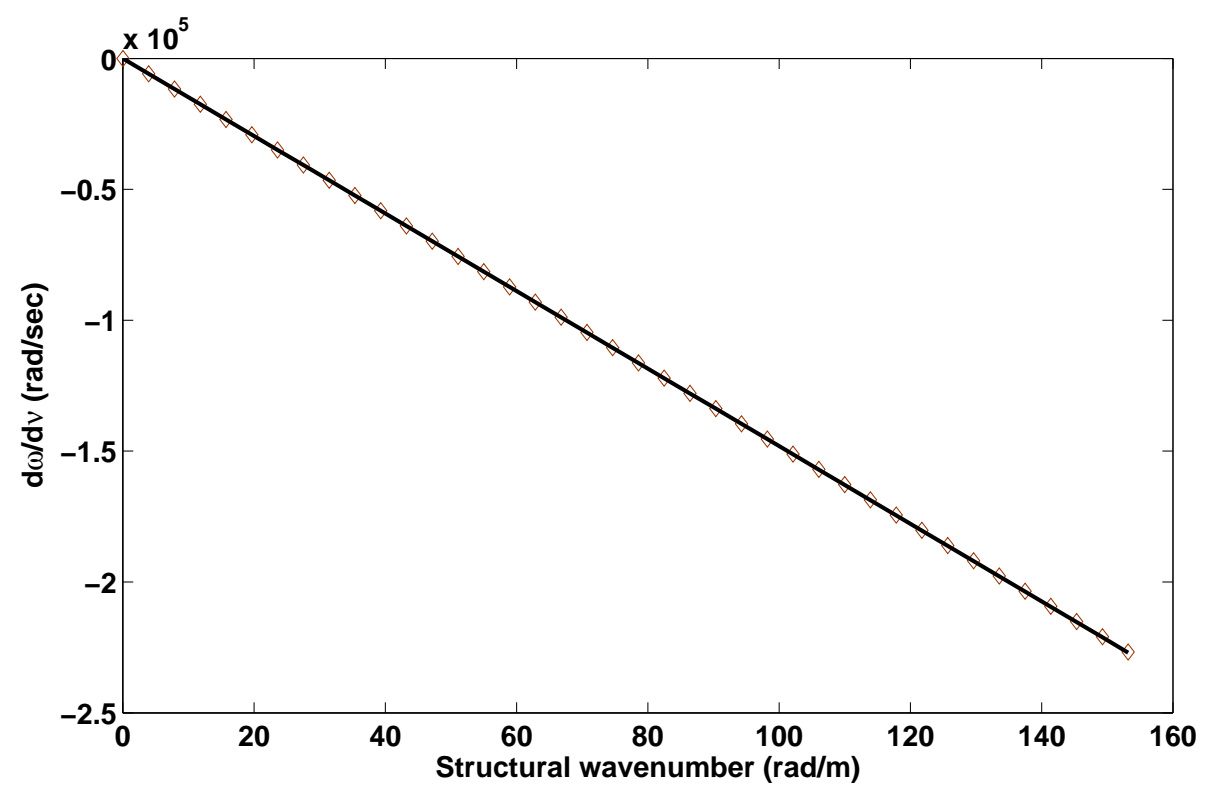

Figure 8: Sensitivity of the angular frequency $\omega$ at which the set of $\varepsilon_{x}, \varepsilon_{y}$ occur under a perturbation of the Poisson's ratio of the structure for the first shear wave type: Analytical solution $(\diamond)$, Numerical calculation $(-)$ 


\subsection{Sandwich panel}

A sandwich panel comprising two facesheets and a core is modelled in this section. The lower facesheet has a thickness $h_{f l}=0.001 \mathrm{~m}$ and is made of a material with $\rho_{f l}=3000 \mathrm{~kg} / \mathrm{m}^{3}, E_{f l}=70 \mathrm{GPa}$ and $v_{f l}=0.1$. The upper facesheet has a thickness equal to $h_{f u}=0.002 \mathrm{~m}$ and is made of the same material as the lower facesheet. The core has a thickness $h_{c}=0.01 \mathrm{~m}$ and is made of a material with $\rho_{c}=50 \mathrm{~kg} / \mathrm{m}^{3}, E_{c}=0.07 \mathrm{GPa}$ and $v_{c}=0.4$. Three FE are used in the sense of thickness in order to model the structure. For the sake of brevity and because of the fact that the out of plane waves are usually the ones that are of interest for vibroacoustic, health monitoring and energy harvesting applications -carrying most of the vibrational energy-, only results on the flexural wave type are presented for the sandwich structure below. The results are compared to a FD approach throughout this section. In order to implement the FD approach a perturbation of $0.001 \%$ was considered for each structural parameter. The resulting FD sensitivity can be computed by

$$
\frac{\partial \omega}{\partial \beta}=\frac{\omega_{p}-\omega_{0}}{\beta_{p}-\beta_{0}}
$$

with $\omega_{p}$ the perturbated angular frequency at which a certain wavenumber value occurs for $\beta_{p}$ and $\omega_{0}$ the corresponding angular frequency for $\beta_{0}$. The perturbation of $\omega$ with respect to $E_{f l}, E_{f u}$ of the structure is presented in Fig.9. It is observed that the increase of both values will stiffen the sandwich structure. For the lower wavenumber values this increase in stiffness will be similar for $\delta E_{f l}$ and $\delta E_{f u}$. However in higher frequencies it can be seen that increasing $E_{f u}$ for the thicker upper facesheet will have a much greater impact (up to $400 \%$ greater) than the increase of $E_{f l}$. The perturbation of 


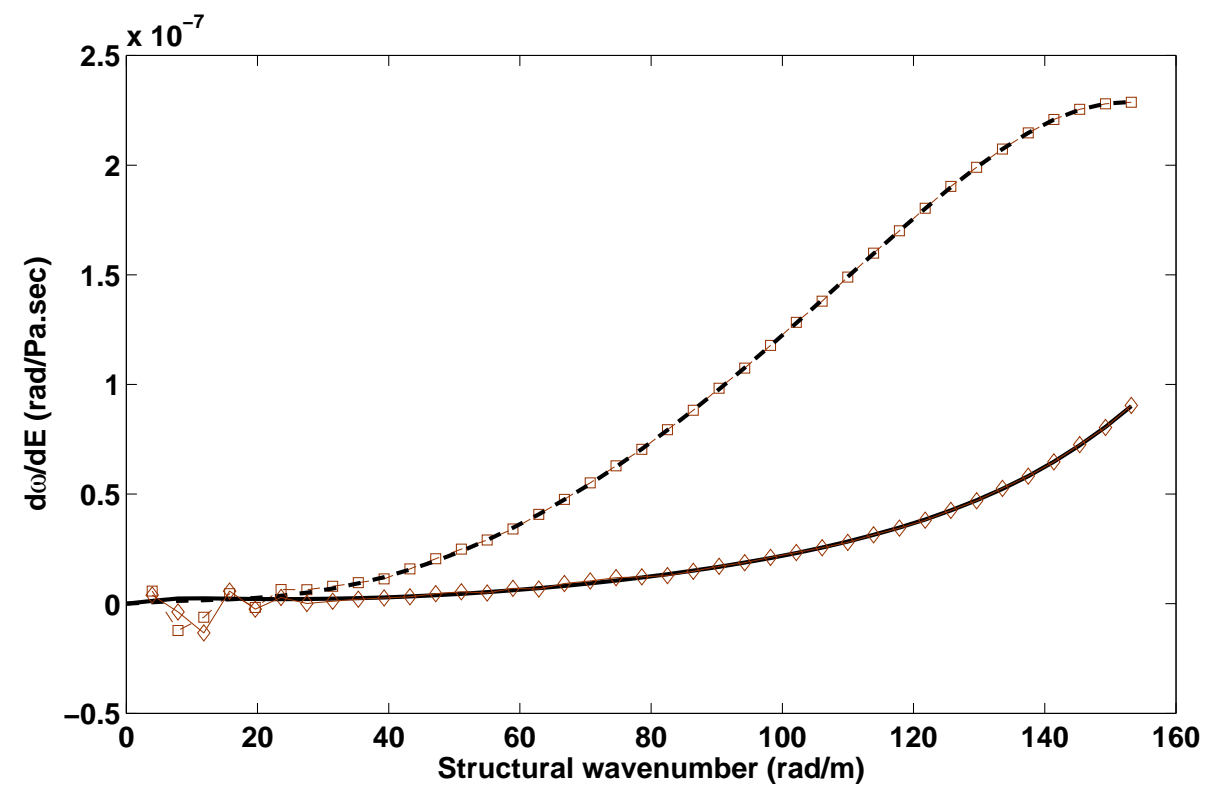

Figure 9: Sensitivity of the angular frequency $\omega$ at which the set of $\varepsilon_{x}, \varepsilon_{y}$ occur under a perturbation of $E$ of the sandwich facesheets for the first flexural wave type of a layered structure: Presented approach for $E_{f l}(-)$, FD computation for $E_{f l}(\diamond)$, Presented approach for $E_{f u}(--)$, FD computation for $E_{f u}(\square)$ 


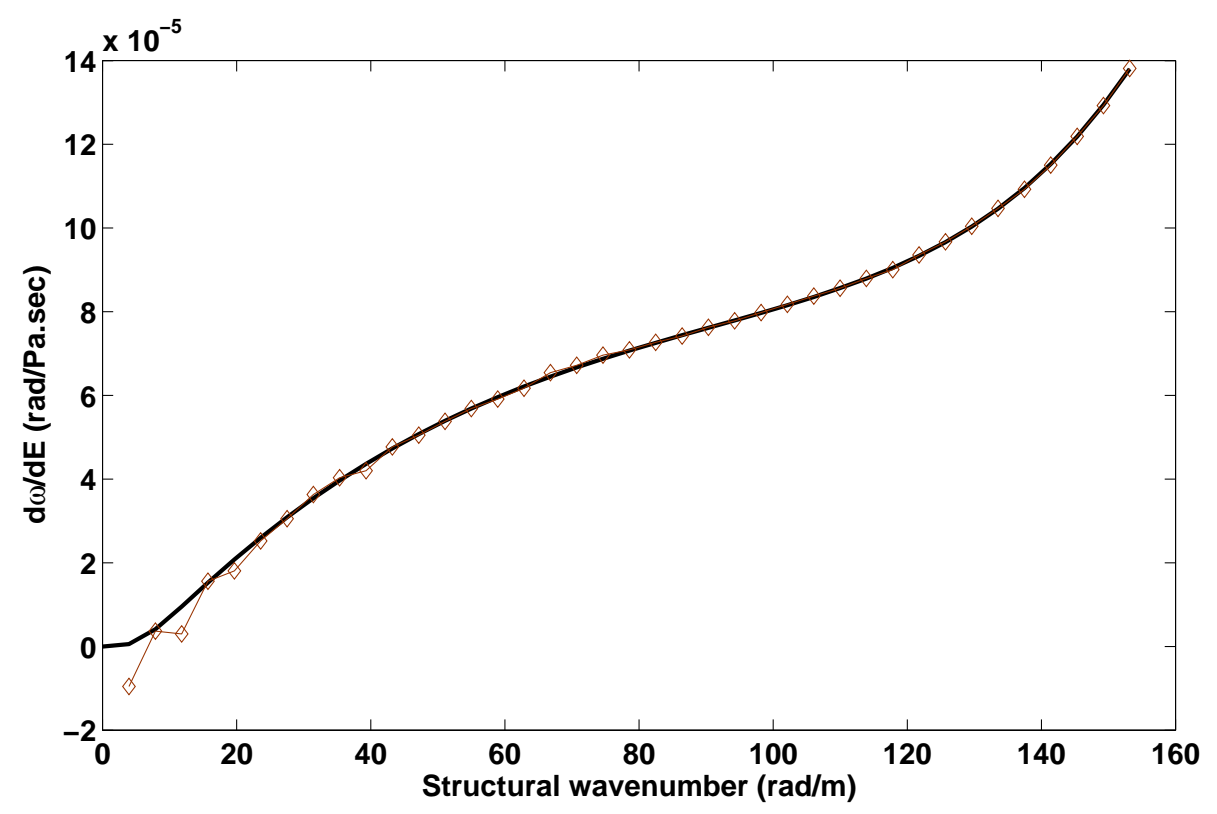

Figure 10: Sensitivity of the angular frequency $\omega$ at which the set of $\varepsilon_{x}, \varepsilon_{y}$ occur under a perturbation of $E_{c}$ for the first flexural wave type of a layered structure: Presented approach $(-)$, FD computation $(\diamond)$

$\omega$ with respect to $E_{c}$ is presented in Fig.10. A fluctuation of the FD results is observed again for low wavenumber values however the results are in very good agreement for higher frequencies. Again it is observed that the effect of $\delta E_{c}$ on the flexural wavenumber varies significantly with respect to the wavenumber value. The perturbation of $\omega$ with respect to $h_{f l}, h_{f u}$ of the structure is presented in Fig.11. It is particularly interesting to note that for lower wavenumbers increasing the thickness of both facesheets will imply a softening effect to the structural behaviour, shifting the flexural wavenumbers to lower frequencies. This mainly suggests that the effect of the added mass overcomes the effect of added stiffness. This effect is similar for both $\delta h_{f l}$ and $\delta h_{f u}$ for low wavenumber values however at higher frequencies the results 


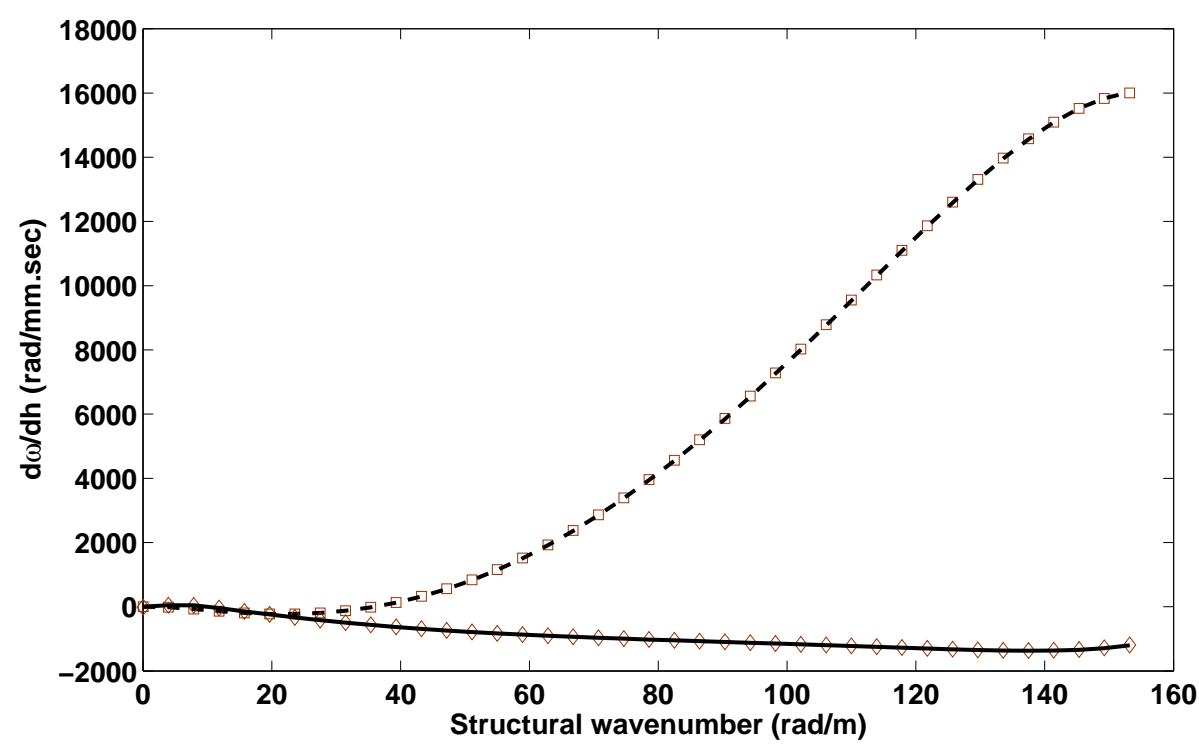

Figure 11: Sensitivity of the angular frequency $\omega$ at which the set of $\varepsilon_{x}, \varepsilon_{y}$ occur under a perturbation of the thickness of the sandwich facesheets for the first flexural wave type of a layered structure: Presented approach for $h_{f l}(-)$, FD computation for $h_{f l}(\diamond)$, Presented approach for $h_{f u}(--)$, FD computation for $h_{f u}(\square)$

change radically for the thicker upper facesheet, with $\delta h_{f u}$ now shifting the wavenumbers to higher frequencies. Again excellent agreement is observed between the presented approach and the FD method. The perturbation of $\omega$ with respect to $h_{c}$ of the sandwich is presented in Fig.12. A very interesting effect is that the influence of $\delta h_{c}$ on the flexural wavenumber becomes maximum at a certain wavenumber value (approximately for $k=100 \mathrm{rad} / \mathrm{m}$ ). A constant decrease of this influence is observed beyond that point. The stiffening effect is probably due to the greater separation of the two facesheets with $\delta h_{c}$. It is very probable however that for higher wavenumber values $\delta h_{c}$ will have a softening effect on the flexural wavenumber with the depicted curve passing to negative values for $\delta \omega$. The perturbation of $\omega$ with respect to $v_{f l}$, 


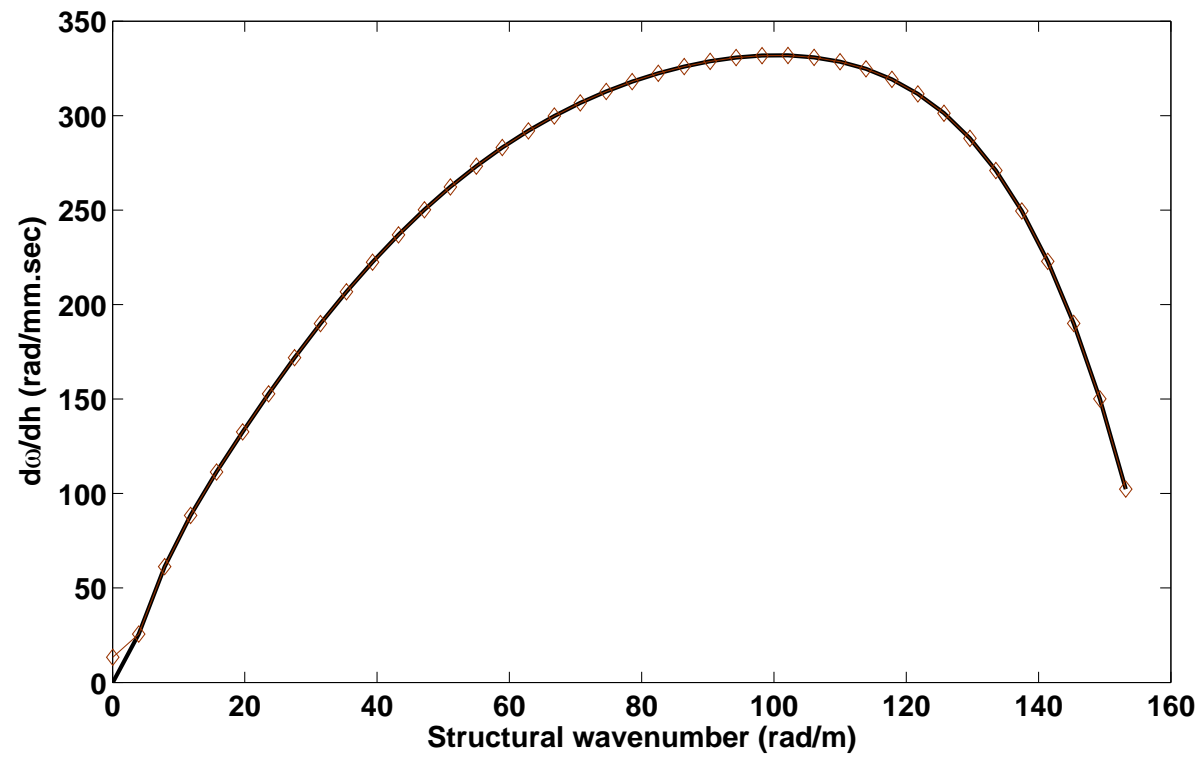

Figure 12: Sensitivity of the angular frequency $\omega$ at which the set of $\varepsilon_{x}, \varepsilon_{y}$ occur under a perturbation of $h_{c}$ for the first flexural wave type of a layered structure: Presented approach (-), FD computation $(\diamond)$ 


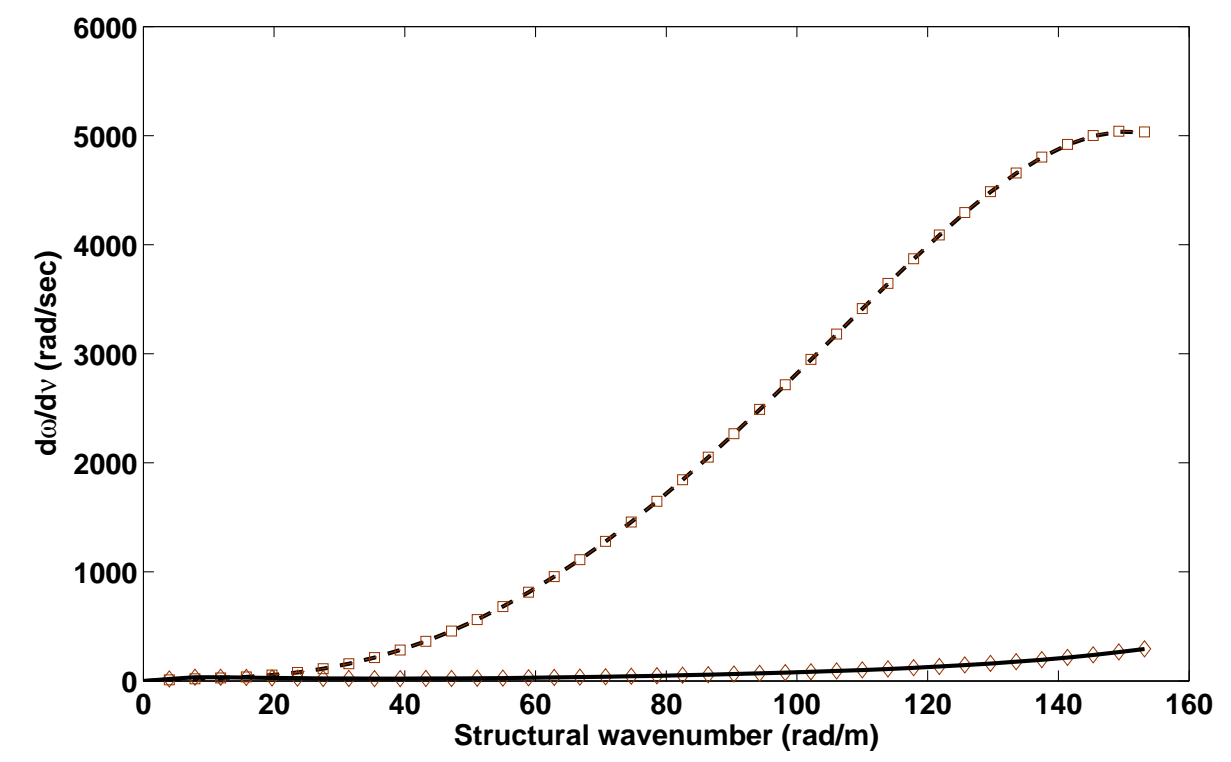

Figure 13: Sensitivity of the angular frequency $\omega$ at which the set of $\varepsilon_{x}, \varepsilon_{y}$ occur under a perturbation of the Poisson's ratio of the sandwich facesheets for the first flexural wave type of a layered structure: Presented approach for $v_{f l}(-)$, FD computation for $v_{f l}(\diamond)$, Presented approach for $v_{f u}(--)$, FD computation for $v_{f u}(\square)$

$v_{f u}$ of the structure is presented in Fig.13. In both cases it can be observed that increasing $v$ for the facesheets will shift the flexural wavenumber curve towards higher frequencies. This phenomenon however will be much more intense for $\delta v_{f u}$ especially at higher wavenumber values. Again excellent agreement is observed between the FD results and the presented approach. The perturbation of $\omega$ with respect to $v_{c}$ for the sandwich core is presented in Fig.14. The effect of $\delta v_{c}$ is softening up to a certain wavenumber value, beyond which a sudden exponential increase is observed which stiffens the flexural structural behaviour. The perturbation of $\omega$ with respect to $\rho_{f l}$ and $\rho_{f u}$ is presented in Fig.15. As expected both $\delta \rho_{f l}$ and $\delta \rho_{f u}$ will shift the wavenumber curve to lower frequencies. This effect will be greater for the 


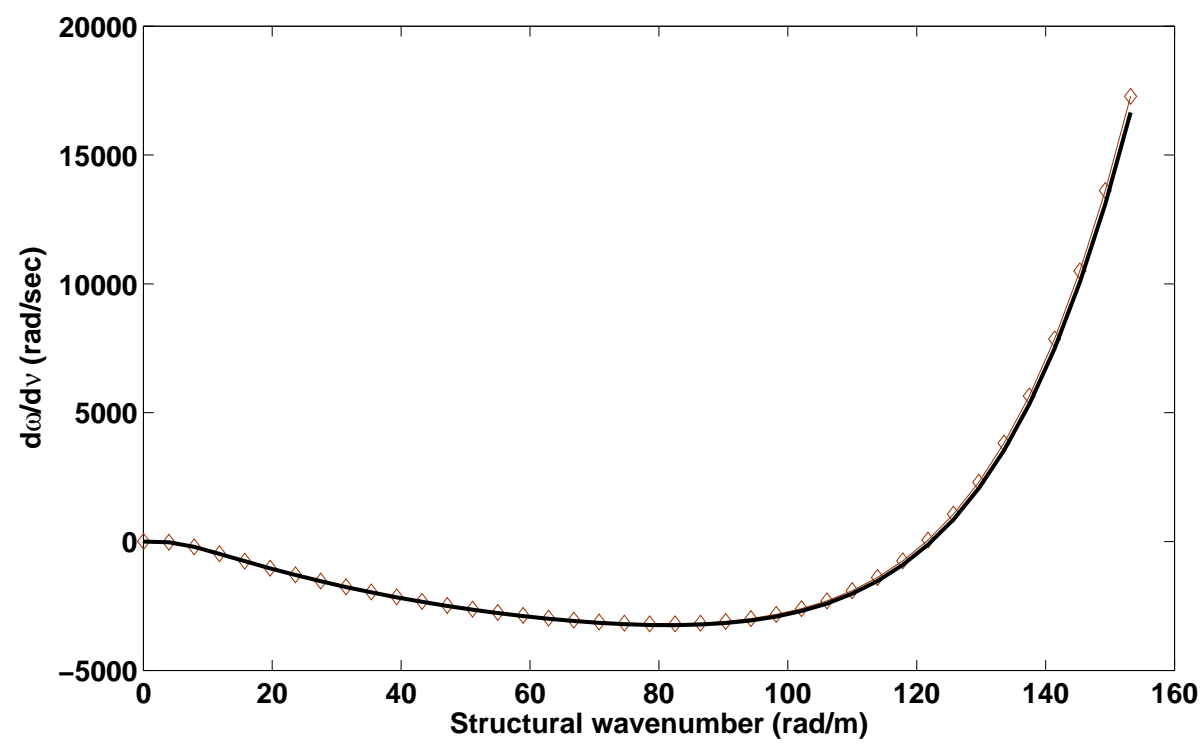

Figure 14: Sensitivity of the angular frequency $\omega$ at which the set of $\varepsilon_{x}, \varepsilon_{y}$ occur under a perturbation of $v_{c}$ for the first flexural wave type of a layered structure: Presented approach $(-)$, FD computation $(\diamond)$ 


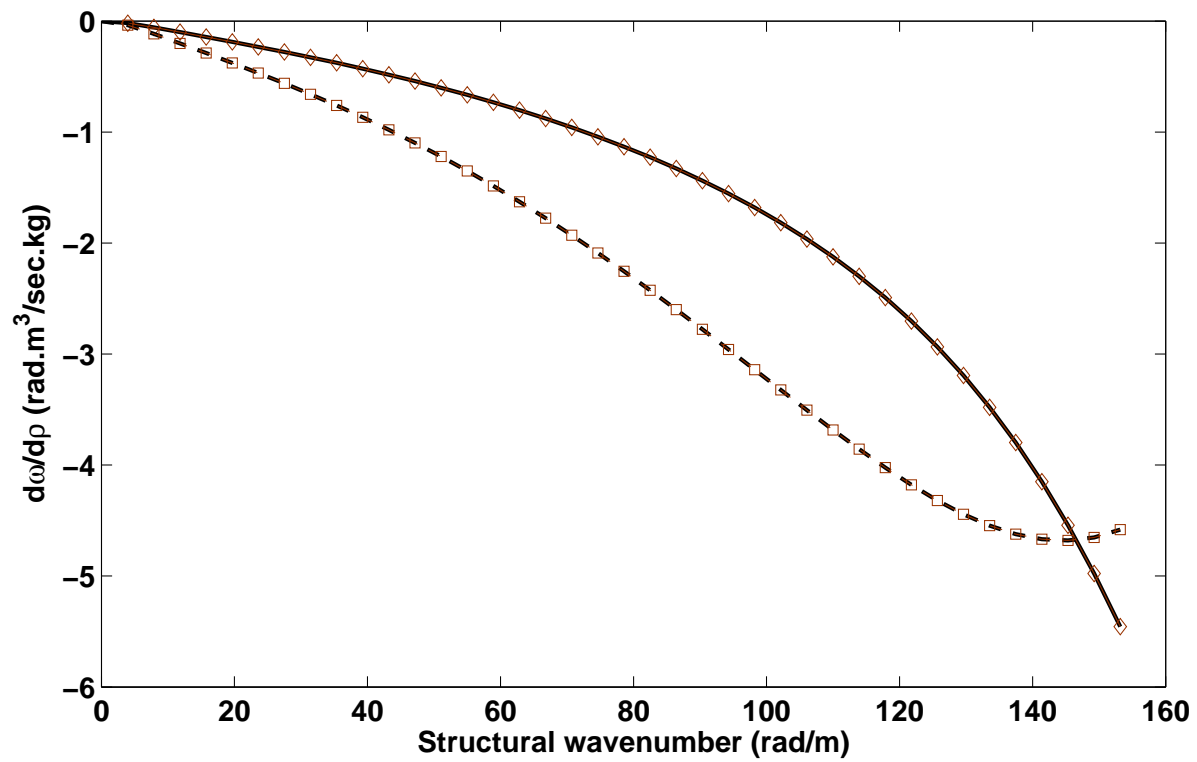

Figure 15: Sensitivity of the angular frequency $\omega$ at which the set of $\varepsilon_{x}, \varepsilon_{y}$ occur under a perturbation of the mass density of the sandwich facesheets for the first flexural wave type: Presented approach for $\rho_{f l}(-)$, FD computation for $\rho_{f l}(\diamond)$, Presented approach for $\rho_{f u}(--)$, FD computation for $\rho_{f u}(\square)$

thicker upper facesheet at low $k$ values. It is very interesting however to see that there is a critical wavenumber value (approximately $k=147 \mathrm{rad} / \mathrm{m}$ ) at which the effect of $\delta \rho_{f l}$ and $\delta \rho_{f u}$ will be the same. Beyond this critical wavenumber the softening effect will be more intense for $\delta \rho_{f l}$ than $\delta \rho_{f u}$. The perturbation of $\omega$ with respect to $\rho_{c}$ for the sandwich core is presented in Fig.16. It is clear that $\delta \rho_{c}$ implies a softening effect to the flexural behaviour of the panel which has an increasing intensity with wavenumber values.

Throughout Fig.9-16, a great wavenumber dependence has been observed for the sensitivity results of the sandwich structure. This exhibits the potential of the presented tool with regard to the optimization of each structural 


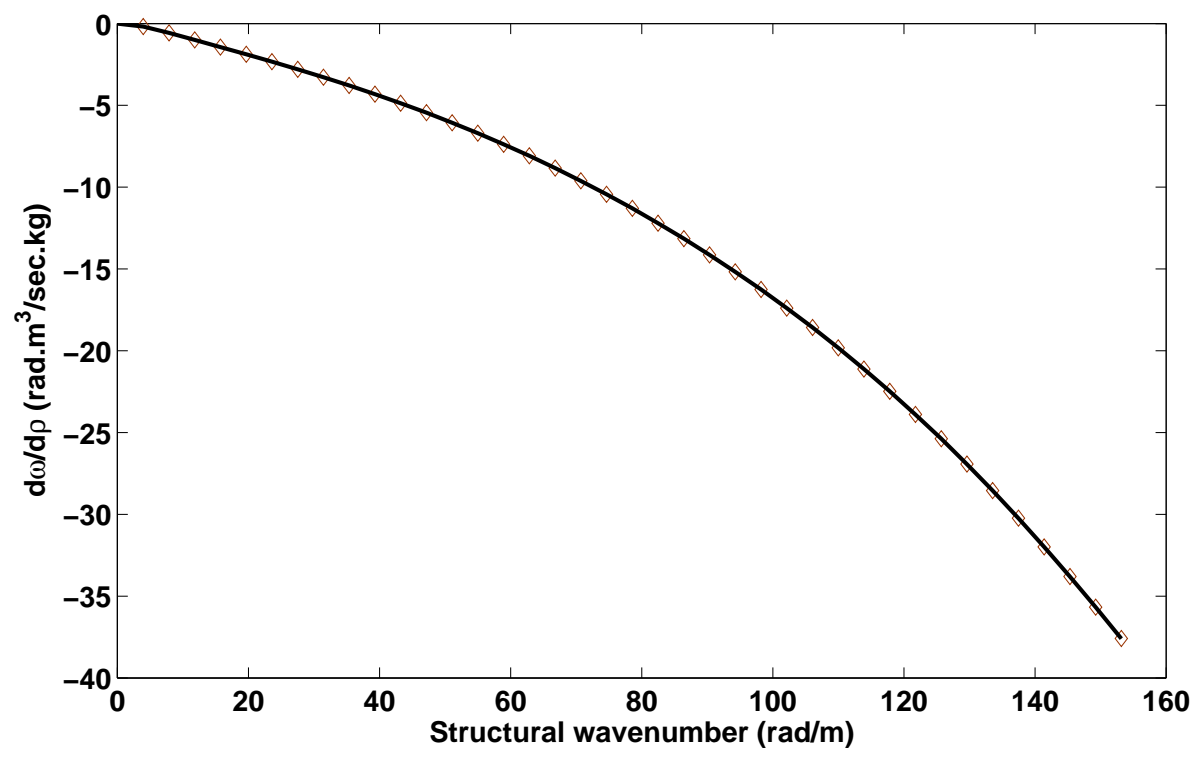

Figure 16: Sensitivity of the angular frequency $\omega$ at which the set of $\varepsilon_{x}, \varepsilon_{y}$ occur under a perturbation of $\rho_{c}$ for the first flexural wave type: Presented approach (-), FD computation $(\diamond)$

layer for specific applications. The model can also be used for computing the effect of the inclusion of smart layers such as auxetics and piezoelectrics or computing the sensitivity of the propagating waves in the presence of damage.

\section{Conclusions}

A numerical continuum-discrete approach for computing the sensitivity of the waves propagating in periodic structures was presented in this paper. The main conclusions of the work are as follows:

(i) A formulation of the two dimensional wave propagation problem was exhibited; this was followed by the derivation of the sensitivity of the waves 
propagating in the periodic structure. The structure can be of arbitrary layering, material characteristics and geometric complexity. The effect of local parameter variation can also be considered.

(ii) A reduced transfer matrix formulation of the wave propagation analysis in one dimensional periodic media was presented, followed by the derivation of the sensitivity of the wavenumber values. As for the first formulation, the structure can be of arbitrary layering, material characteristics and geometric complexity. This approach however tends to be less computationally efficient due to the involved inversions and multiplications of DSM partitions.

(iii) A monolayer panel was modelled by the presented approach and the resulting sensitivity values were validated through the CPT.

(iv) Moreover, an asymmetric sandwich structure was also modelled. The sensitivity of the propagating flexural wave with respect to the structural parameters of the facesheets and the core were computed and the results were successfully compared to the ones provided by the FD method.

(v) A great wavenumber dependence has been observed for the sensitivity results of the sandwich structure. This exhibits the potential of the presented tool with regard to providing an efficient and robust optimization scheme for layered structures, tailored for specific applications. The model can also be used for computing the effect of the inclusion of smart layers such as auxetics and piezoelectrics or computing the sensitivity of the propagating waves in the presence of damage.

(vi) The formulation of the symbolic expression of the stiffness and mass matrices for a linear solid FE were also presented. These formulations can be used in order to efficiently deduce the symbolic global matrices of the 
modelled segment, as well as the sensitivity of the global matrices with regard to any structural parameter.

\section{References}

[1] D. J. Mead, A general theory of harmonic wave propagation in linear periodic systems with multiple coupling, Journal of Sound and Vibration 27 (1973) 235-60.

[2] R. Langley, A note on the force boundary conditions for two-dimensional periodic structures with corner freedoms, Journal of Sound and Vibration 167 (1993) 377-81.

[3] W. Zhong, F. Williams, On the direct solution of wave propagation for repetitive structures, Journal of Sound and Vibration 181 (1995) $485-501$.

[4] B. R. Mace, D. Duhamel, M. J. Brennan, L. Hinke, Finite element prediction of wave motion in structural waveguides, The Journal of the Acoustical Society of America 117 (2005) 2835-43.

[5] J.-M. Mencik, M. Ichchou, Multi-mode propagation and diffusion in structures through finite elements, European Journal of MechanicsA/Solids 24 (2005) 877-98.

[6] D. Duhamel, B. R. Mace, M. J. Brennan, Finite element analysis of the vibrations of waveguides and periodic structures, Journal of Sound and Vibration 294 (2006) 205-20. 
[7] J.-M. Mencik, On the low-and mid-frequency forced response of elastic structures using wave finite elements with one-dimensional propagation, Computers \& Structures 88 (2010) 674-89.

[8] J. M. Renno, B. R. Mace, On the forced response of waveguides using the wave and finite element method, Journal of Sound and Vibration 329 (2010) 5474-88.

[9] B. Mace, E. Manconi, Modelling wave propagation in two-dimensional structures using finite element analysis, Journal of Sound and Vibration 318 (2008) 884-902.

[10] J. Renno, B. Mace, Calculating the forced response of two-dimensional homogeneous media using the wave and finite element method, Journal of Sound and Vibration 330 (2011) 5913-27.

[11] D. Chronopoulos, B. Troclet, M. Ichchou, J. Laine, A unified approach for the broadband vibroacoustic response of composite shells, Composites Part B: Engineering 43 (2012) 1837-46.

[12] D. Chronopoulos, B. Troclet, O. Bareille, M. Ichchou, Modeling the response of composite panels by a dynamic stiffness approach, Composite Structures 96 (2013) 111-20.

[13] D. Chronopoulos, M. Ichchou, B. Troclet, O. Bareille, Efficient prediction of the response of layered shells by a dynamic stiffness approach, Composite Structures 97 (2013) 401-4.

[14] D. Chronopoulos, M. Ichchou, B. Troclet, O. Bareille, Predicting the broadband vibroacoustic response of systems subject to aeroacoustic 
loads by a krylov subspace reduction, Applied Acoustics 74 (2013) 1394405.

[15] D. Chronopoulos, M. Ichchou, B. Troclet, O. Bareille, Thermal effects on the sound transmission through aerospace composite structures, Aerospace Science and Technology 30 (2013) 192-9.

[16] D. Chronopoulos, M. Ichchou, B. Troclet, O. Bareille, Predicting the broadband response of a layered cone-cylinder-cone shell, Composite Structures 107 (2014) 149-59.

[17] D. Chronopoulos, M. Ichchou, B. Troclet, O. Bareille, Computing the broadband vibroacoustic response of arbitrarily thick layered panels by a wave finite element approach, Applied Acoustics 77 (2014) 89-98.

[18] V. Polenta, S. Garvey, D. Chronopoulos, A. Long, H. Morvan, Optimal internal pressurisation of cylindrical shells for maximising their critical bending load, Thin-Walled Structures 87 (2015) 133-8.

[19] T. Ampatzidis, D. Chronopoulos, Acoustic transmission properties of pressurised and pre-stressed composite structures, Composite Structures 152 (2016) 900-12.

[20] I. Antoniadis, D. Chronopoulos, V. Spitas, D. Koulocheris, Hyperdamping properties of a stiff and stable linear oscillator with a negative stiffness element, Journal of Sound and Vibration 346 (2015) 37-52.

[21] D. Chronopoulos, M. Collet, M. Ichchou, Damping enhancement of composite panels by inclusion of shunted piezoelectric patches: A wavebased modelling approach, Materials 8 (2015) 815-28. 
[22] D. Chronopoulos, I. Antoniadis, M. Collet, M. Ichchou, Enhancement of wave damping within metamaterials having embedded negative stiffness inclusions, Wave Motion 58 (2015) 165-79.

[23] D. Chronopoulos, Design optimization of composite structures operating in acoustic environments, Journal of Sound and Vibration 355 (2015) $322-44$.

[24] M. Ben Souf, D. Chronopoulos, M. Ichchou, O. Bareille, M. Haddar, On the variability of the sound transmission loss of composite panels through a parametric probabilistic approach, Journal of Computational Acoustics 24 (2016).

[25] D. Chronopoulos, Wave steering effects in anisotropic composite structures: Direct calculation of the energy skew angle through a finite element scheme, Ultrasonics 73 (2017) 43-8.

[26] R. B. Nelson, Simplified calculation of eigenvector derivatives, AIAA journal 14 (1976) 1201-5.

[27] R. T. Haftka, H. M. Adelman, Recent developments in structural sensitivity analysis, Structural optimization 1 (1989) 137-51.

[28] S. Adhikari, M. I. Friswell, Eigenderivative analysis of asymmetric nonconservative systems, International Journal for Numerical Methods in Engineering 51 (2001) 709-33.

[29] K. K. Choi, N.-H. Kim, Structural sensitivity analysis and optimization 1: linear systems, volume 1, Springer, 2006. 
[30] K. Sobczyk, Stochastic wave propagation, Elsevier, 1985.

[31] A. Belyaev, Comparative study of various approaches to stochastic elastic wave propagation, Acta mechanica 125 (1997) 3-16.

[32] K. Koo, B. Pluymers, W. Desmet, S. Wang, Vibro-acoustic design sensitivity analysis using the wave-based method, Journal of Sound and Vibration 330 (2011) 4340-51.

[33] M. Ruzzene, F. Scarpa, Directional and band-gap behavior of periodic auxetic lattices, physica status solidi (b) 242 (2005) 665-80.

[34] M. Ichchou, F. Bouchoucha, M. Ben Souf, O. Dessombz, M. Haddar, Stochastic wave finite element for random periodic media through firstorder perturbation, Computer Methods in Applied Mechanics and Engineering 200 (2011) 2805-13.

[35] B. Souf, O. Bareille, M. Ichchou, F. Bouchoucha, M. Haddar, Waves and energy in random elastic guided media through the stochastic wave finite element method, Physics Letters A 377 (2013) 2255-64.

[36] V. Cotoni, R. S. Langley, P. J. Shorter, A statistical energy analysis subsystem formulation using finite element and periodic structure theory, Journal of Sound and Vibration 318 (2008) 1077-108.

[37] S. Finnveden, Evaluation of modal density and group velocity by a finite element method, Journal of Sound and Vibration 273 (2004) 51-75.

[38] C. A. Felippa, R. W. Clough, The finite element method in solid mechanics, American Mathematical Society, 1970. 


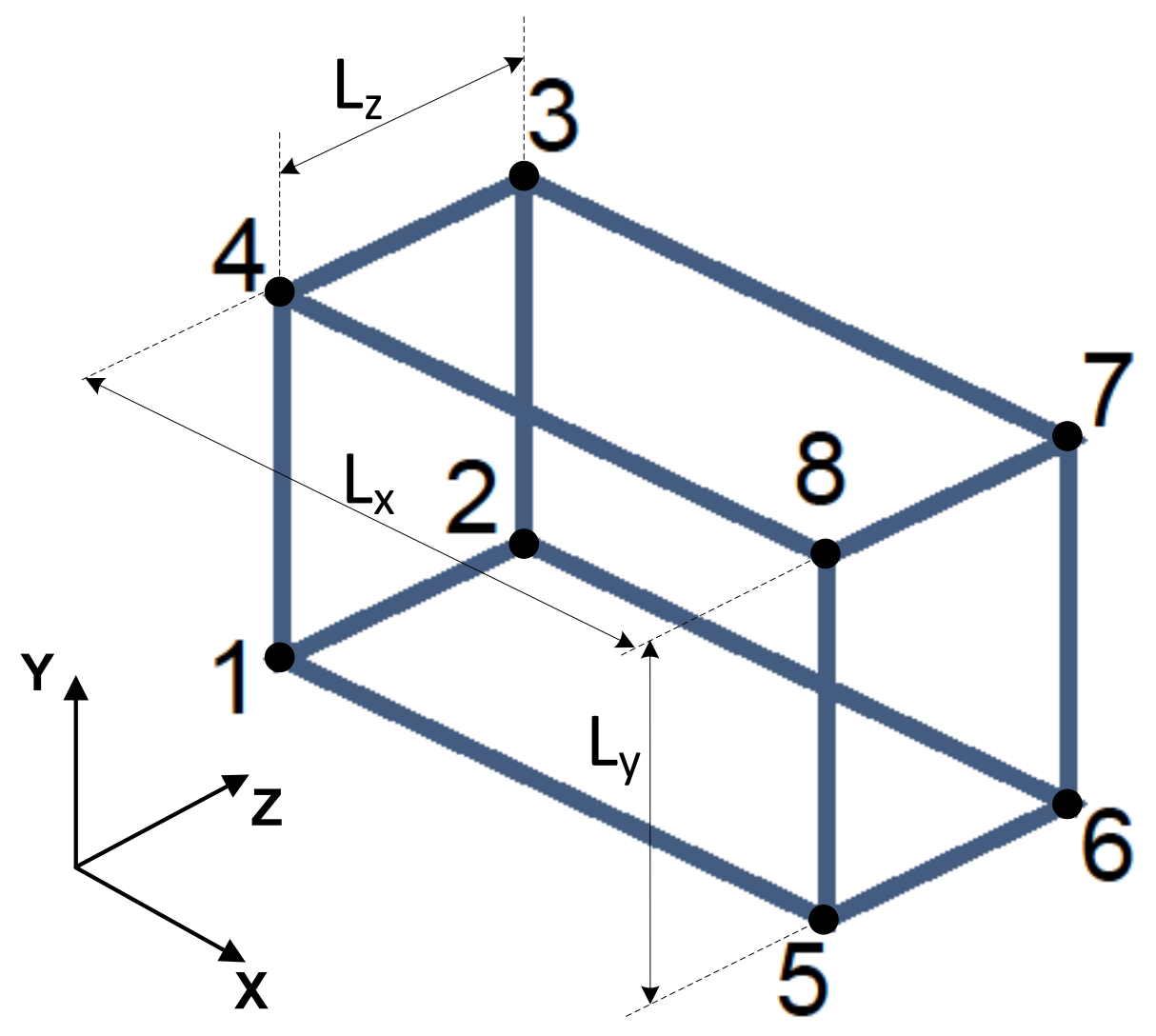

Figure 17: The considered solid FE

Appendix A. Sensitivity analysis of a solid FE

A linear solid FE is hereby considered as shown in Fig.17. Following the isoparametric notation introduced in [38] the geometry of the element is 
described as

$$
\left\{\begin{array}{l}
x \\
y \\
z
\end{array}\right\}=\left[\begin{array}{cccccccc}
x_{1} & x_{2} & x_{3} & x_{4} & x_{5} & x_{6} & x_{7} & x_{8} \\
y_{1} & y_{2} & y_{3} & y_{4} & y_{5} & y_{6} & y_{7} & y_{8} \\
z_{1} & z_{2} & z_{3} & z_{4} & z_{5} & z_{6} & z_{7} & z_{8}
\end{array}\right]\left\{\begin{array}{c}
N_{1} \\
N_{2} \\
N_{3} \\
N_{4} \\
N_{5} \\
N_{6} \\
N_{7} \\
N_{8}
\end{array}\right\}
$$

The displacement interpolations are expressed as

$$
\left\{\begin{array}{l}
u_{x} \\
u_{y} \\
u_{z}
\end{array}\right\}=\left[\begin{array}{cccccccc}
u_{x 1} & u_{x 2} & u_{x 3} & u_{x 4} & u_{x 5} & u_{x 6} & u_{x 7} & u_{x 8} \\
u_{y 1} & u_{y 2} & u_{y 3} & u_{y 4} & u_{y 5} & u_{y 6} & u_{y 7} & u_{y 8} \\
u_{z 1} & u_{z 2} & u_{z 3} & u_{z 4} & u_{z 5} & u_{z 6} & u_{z 7} & u_{z 8}
\end{array}\right]\left\{\begin{array}{c}
N_{1} \\
N_{2} \\
N_{3} \\
N_{4} \\
N_{5} \\
N_{6} \\
N_{7} \\
N_{8}
\end{array}\right\}
$$


Linear shape functions are assumed for the element

$$
\begin{aligned}
& N_{1}=\frac{1}{8}(1-\xi)(1-\eta)(1+\mu) \\
& N_{2}=\frac{1}{8}(1-\xi)(1-\eta)(1-\mu) \\
& N_{3}=\frac{1}{8}(1-\xi)(1+\eta)(1-\mu) \\
& N_{4}=\frac{1}{8}(1-\xi)(1+\eta)(1+\mu) \\
& N_{5}=\frac{1}{8}(1+\xi)(1-\eta)(1+\mu) \\
& N_{6}=\frac{1}{8}(1+\xi)(1-\eta)(1-\mu) \\
& N_{7}=\frac{1}{8}(1+\xi)(1+\eta)(1-\mu) \\
& N_{8}=\frac{1}{8}(1+\xi)(1+\eta)(1+\mu)
\end{aligned}
$$

The element stiffness matrix $\mathbf{k}$ is formally given by the volume integral

$$
\mathbf{k}=\int_{-1}^{1} \int_{-1}^{1} \int_{-1}^{1} \mathbf{B}^{\top} \mathbf{D B}|\mathbf{J}| \mathrm{d} \eta \mathrm{d} \xi \mathrm{d} \mu
$$

while the element mass matrix $\mathbf{m}$ can be determined as

$$
\mathbf{m}=\int_{-1}^{1} \int_{-1}^{1} \int_{-1}^{1} \mathbf{N}^{\top} \rho_{m} \mathbf{N}|\mathbf{J}| \mathrm{d} \eta \mathrm{d} \xi \mathrm{d} \mu
$$

with

$$
\mathbf{N}=\left[\begin{array}{ccccccc}
N_{1} & 0 & 0 & \cdots & N_{8} & 0 & 0 \\
0 & N_{1} & 0 & \cdots & 0 & N_{8} & 0 \\
0 & 0 & N_{1} & \cdots & 0 & 0 & N_{8}
\end{array}\right]
$$


while $\rho_{m}$ is the mass density of the material. It is also noted that

$$
\mathbf{B}=\left[\begin{array}{cccccccc}
\frac{\partial N_{1}}{\partial x} & 0 & 0 & \frac{\partial N_{2}}{\partial x} & \cdots & \frac{\partial N_{8}}{\partial x} & 0 & 0 \\
0 & \frac{\partial N_{1}}{\partial y} & 0 & 0 & \cdots & 0 & \frac{\partial N_{8}}{\partial y} & 0 \\
0 & 0 & \frac{\partial N_{1}}{\partial z} & 0 & \cdots & 0 & 0 & \frac{\partial N_{8}}{\partial z} \\
\frac{\partial N_{1}}{\partial y} & \frac{\partial N_{1}}{\partial x} & 0 & \frac{\partial N_{2}}{\partial y} & \cdots & \frac{\partial N_{8}}{\partial y} & \frac{\partial N_{8}}{\partial x} & 0 \\
0 & \frac{\partial N_{1}}{\partial z} & \frac{\partial N_{1}}{\partial y} & 0 & \cdots & 0 & \frac{\partial N_{8}}{\partial z} & \frac{\partial N_{8}}{\partial y} \\
\frac{\partial N_{1}}{\partial z} & 0 & \frac{\partial N_{1}}{\partial x} & \frac{\partial N_{2}}{\partial z} & \cdots & \frac{\partial N_{8}}{\partial z} & 0 & \frac{\partial N_{8}}{\partial x}
\end{array}\right]\left\{\begin{array}{c}
u_{x 1} \\
u_{y 1} \\
u_{z 1} \\
u_{x 2} \\
\cdots \\
u_{x 8} \\
u_{y 8} \\
u_{z 8}
\end{array}\right\}
$$

The Jacobian matrix of the element is

$$
\mathbf{J}=\left[\begin{array}{lll}
\frac{\partial x}{\partial \xi} & \frac{\partial y}{\partial \xi} & \frac{\partial z}{\partial \xi} \\
\frac{\partial x}{\partial \eta} & \frac{\partial y}{\partial \eta} & \frac{\partial z}{\partial \eta} \\
\frac{\partial x}{\partial \mu} & \frac{\partial y}{\partial \mu} & \frac{\partial z}{\partial \mu}
\end{array}\right]
$$

while the the flexibility matrix of the element for an orthotropic material $\mathbf{D}^{-1}$ can generally be written as

$$
\mathbf{D}^{-1}=\left[\begin{array}{cccccc}
\frac{1}{E_{x}} & \frac{-v_{x y}}{E_{x}} & \frac{-v_{x z}}{E_{x}} & 0 & 0 & 0 \\
\frac{-v_{y x}}{E_{y}} & \frac{1}{E_{y}} & \frac{-v_{y z}}{E_{y}} & 0 & 0 & 0 \\
\frac{-v_{z x}}{E_{z}} & \frac{-v_{z y}}{E_{z}} & \frac{1}{E_{z}} & 0 & 0 & 0 \\
0 & 0 & 0 & \frac{1}{G_{x y}} & 0 & 0 \\
0 & 0 & 0 & 0 & \frac{1}{G_{y z}} & 0 \\
0 & 0 & 0 & 0 & 0 & \frac{1}{G_{x z}}
\end{array}\right]
$$

The assumption of the undeformed FE being a rectangular parallelepiped is hereby adopted. The coordinates $x_{1}, x_{2}, x_{3}, x_{4}, x_{5}, x_{6}, x_{7}, x_{8}, y_{1}, y_{2}, y_{3}, y_{4}, y_{5}, y_{6}, y_{7}, y_{8}$, 
and $z_{1}, z_{2}, z_{3}, z_{4}, z_{5}, z_{6}, z_{7}, z_{8}$, can then be replaced by $L_{x}, L_{y}, L_{z}$ in the expression of $\mathbf{B}$. The generic expression for $\mathbf{m}$ is thus given as

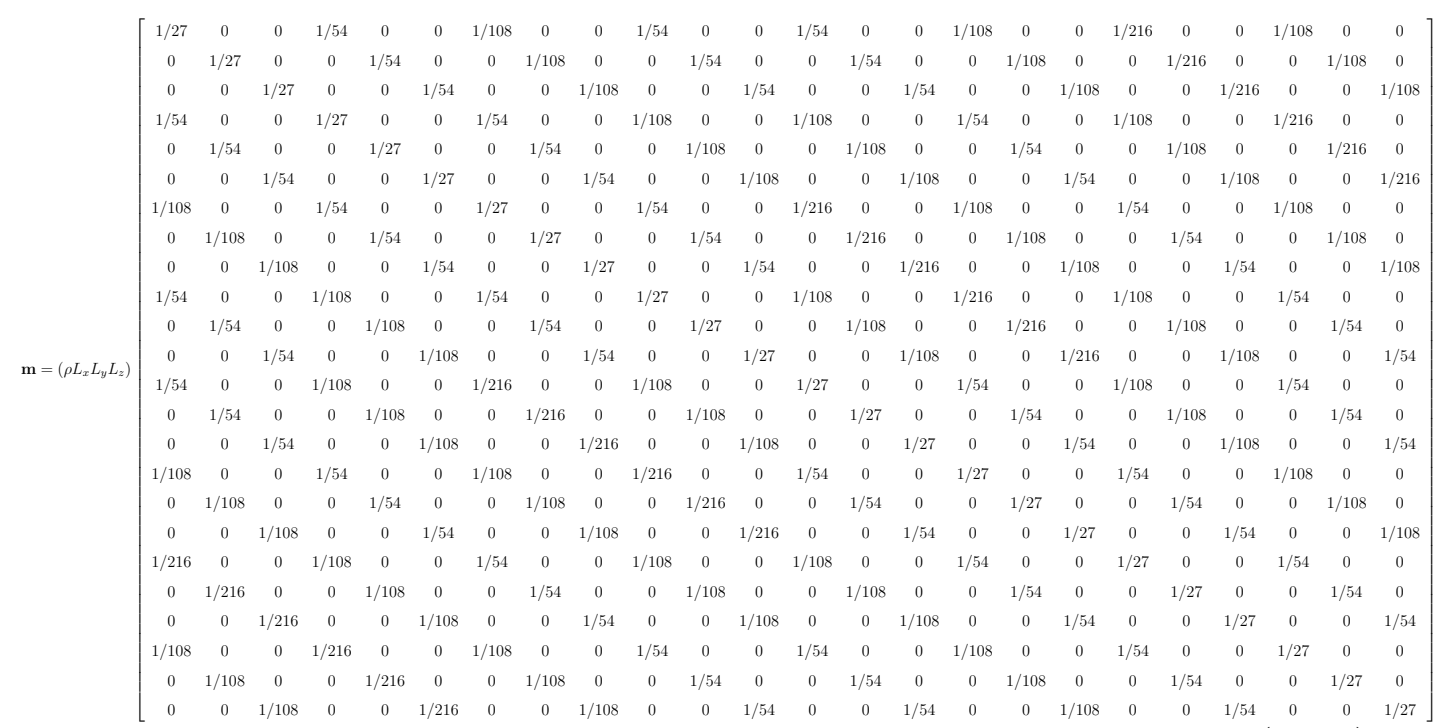

(A.10)

while the symbolic generic expression of $\mathbf{k}$ can be derived exactly in the same way but is hereby intentionally omitted for the sake of brevity.

The generic sensitivity expressions $\frac{\partial \mathbf{k}}{\partial \beta_{i}}, \frac{\partial \mathbf{m}}{\partial \beta_{i}}$ as well as $\frac{\partial^{2} \mathbf{k}}{\partial \beta_{j} \partial \beta_{i}}, \frac{\partial^{2} \mathbf{m}}{\partial \beta_{j} \partial \beta_{i}}$ with $\beta_{i}, \beta_{j}$ being design parameters can therefore be calculated as a function of $E_{x}, E_{y}, E_{z}, v_{x y}, v_{x z}, v_{y z}, G_{x y}, G_{x z}, G_{y z}, L_{x}, L_{y}, L_{z}$ by differentiating over the generic expressions for $\mathbf{k}, \mathbf{m}$. 


\section{List of symbols}

$\Re$

D

$\mathbb{M}, \mathbb{K}$

$\mathrm{T}$

R

$\mathbf{m}, \mathbf{k}$

f

q

$\mathrm{x}$

$\mathbf{x}_{0 i}$

$\mathbf{y}_{0 i}$

$\mathbf{z}_{0 i}$

$B, T, L, R, I$

E

$L_{x}, L_{y}$

$h$

$k$

$l_{x}, l_{y}$

$s$

$t$

$x, y$

$\beta$

$\gamma$

$\epsilon$

$\varepsilon$

$\lambda$

$v$

$\rho$

$\omega$
Real operator

Dynamics Stiffness Matrix (DSM) of a waveguide's modelled periodic se Mass and stiffness matrices of the modelled segment

Wave propagation transfer matrix

Transformation matrix

Mass and stiffness matrices of a single FE

Forcing vector for an elastic waveguide

Physical displacement vector for an elastic waveguide

Reduced set of DoF

Unperturbed eigenvector of the DSM

Unperturbed left eigenvector of wave transfer matrix $\mathbf{T}$

Unperturbed right eigenvector of wave transfer matrix $\mathbf{T}$

Bottom, Top, Left, Right sides and Internal indices

Material Young's modulus

Dimensions of the entire panel

Thickness

Wavenumber

Dimensions of the modelled periodic structural segment

Periodic segment positioning index

Time

Propagation directions

Design variable

Propagation constant and eigenvalue of wave transfer matrix $\mathbf{T}$ 45

Perturbation coefficient

Propagation constant

Eigenvalue of the DSM

Poisson's ratio

Material density

Angular frequency 


\section{List of abbreviations}

$\begin{array}{ll}\text { CPT } & \text { Classical Plate Theory } \\ \text { DoF } & \text { Degree of Freedom } \\ \text { DSM } & \text { Dynamic Stiffness Matrix } \\ \text { FD } & \text { Finite Differences } \\ \text { FE } & \text { Finite Element } \\ \text { PST } & \text { Periodic Structure Theory } \\ \text { WFE } & \text { Wave and Finite Element }\end{array}$

